\title{
Cumulant expansion of the periodic Anderson model: General derivation
}

\author{
M. S. Figueira \\ Instituto de Física, Universidade Federal Fluminense, 24000-970 Niterói, Rio de Janeiro, Brazil \\ M. E. Foglio \\ Instituto de Física "Gleb Wataghin," Universidade Estadual de Campinas, 13083-970 Campinas, São Paulo, Brazil \\ G. G. Martinez \\ Instituto de Física, Universidade Federal do Rio Grande do Sul, 91501-970 Porto Alegre, Rio Grande do Sul, Brazil
}

(Received 29 December 1993; revised manuscript received 21 September 1994)

\begin{abstract}
We extend to the periodic Anderson model (PAM) the diagrammatic expansion in cumulants that was employed by Hubbard to study his model of a narrow band of strongly correlated electrons. The PAM is a lattice of localized and strongly correlated electrons with spin one-half and without orbital degeneracy, hybridized with a wide band of uncorrelated conduction electrons. We have extended the model by considering localized electronic states with an arbitrary scheme of energy levels: this extension would be useful to study intermediate valence compounds of Eu or Tm with the present formalism. We give the rules for the diagrammatic calculation of the grand canonical potential and of the Green's functions for the general model: only connected diagrams appear in those calculations and the lattice sums are unrestricted. To generate the cumulant averages it was necessary to employ external fields $\xi$ that are Grassmann variables. We have found a simple way to extend the diagrammatic rules to the $\xi \neq 0$ case. The absence of excluded site restrictions, that leads to complicated excluded volume problems in other treatments, and the existence of linked cluster expansions, are features of the cumulant expansion. As an application of the present method, we have calculated the occupation numbers of localized and conduction electrons for the PAM in the limit of infinite Coulomb repulsion $(U \rightarrow \infty)$.
\end{abstract}

\section{INTRODUCTION}

Since the early days of quantum mechanics, the importance of strong correlations was recognized, and the Heitler-London model of the hydrogen molecule gave a better overall description than the competing method of molecular orbitals because this effect is built into the wave function. The simplicity of the second method permitted a greater application to many problems, and although the importance of the strong correlation was not forgotten, it is only with the fairly recent discovery of phenomena like intermediate valence, Kondo systems, and particularly the high- $T c$ superconductivity, that the study of strong correlations in solid state systems became a pressing subject. A deceptively simple model was introduced by Hubbard, ${ }^{1}$ for the narrow-band limit, and at present there are exact solutions only for very particular values of the parameters or dimensions of the system. For this reason, it seems valuable to explore approximate methods, and this is the main motivation of the present work. In the fifth paper of the series, ${ }^{2}$ Hubbard discussed cumulant expansions around the atomic limit, a technique that had already been applied ${ }^{3-6}$ to a different type of "localized" system, such as the Ising and Heisenberg models. To describe the localized electronic states, Hubbard introduced $X$ operators: $X_{j b a}=|j b\rangle\langle j a|$ transforms the local state $|a\rangle$ at site $j$ into the local state $|b\rangle$ at the same site. With these operators one can include all the local correlations into a very simple unperturbed Hamiltonian, and the hopping term becomes the perturbation. An important aspect of these operators is that it is very simple to write an unperturbed Hamiltonian that describes a subset of the ionic states at a given site, and one could extend the calculations of rather simplified models like the one treated here, to systems that describe more realistic systems. For this reason we shall follow rather closely Hubbard's treatment of the problem, rather than considering only Green's functions (GF) that correspond to the usual electronic Fermi operators, as done by Metzner. ${ }^{7}$ The $X$ operators are neither bosons nor fermions, and Wick's theorem is not valid, but Hubbard developed a diagrammatic calculation, and was able to prove the validity of a "linkedcluster expansion," which involves only unrestricted lattice sums of connected diagrams. The technique was not very much explored by its author, but several papers that study the Hubbard model by this method have recently appeared. ${ }^{7-9}$

The periodic Anderson model (PAM) is an extension of the Hubbard model that is believed to describe in a schematic way the anomalous behavior of several rare earth compounds that are intermediate valence or Kondo systems. This subject has received much attention in recent years: there are several recent review articles devoted to this problem. ${ }^{10}$

The main objective of the present paper is to extend the cumulant expansions around the atomic limit of the Hubbard model ${ }^{2}$ to the PAM and to a generalization of the PAM that considers a lattice of localized systems with an arbitrary system of levels, and in Sec. II we write 
its Hamiltonian employing Hubbard's $X$ operators. The reason for studying this general model is that several of the rare earth ions that present the anomalous behavior (e.g., Eu and Tm) have a series of levels, relevant to the properties of interest, that cannot be described with the simple PAM. Although the applications of a following paper refer mainly to the PAM, the properties derived here are valid for the general model.

The correlations are very effective in the rare earth systems because the $f$ electrons are strongly localized in those ions, and although one can apply the present treatment to systems with different localized electrons, we shall refer to them as " $f$ electrons." In the usual PAM one neglects the width of the $f$ electrons of the Hubbard model, and adds a wide band of conduction electrons (called $c$ electrons in what follows), which hybridize with the localized $f$ electrons. One then wonders why the linked cluster theorem, which was valid in the Hubbard model because the unperturbed Hamiltonian is one of localized electrons, remains true when the extended conduction electrons are added to the problem. The answer lies in the fact that any cumulant average that contains two or more statistically independent variables is zero. ${ }^{11}$ In the absence of hybridization, a localized electron at a given site is statistically independent both of all the localized electrons at other sites and of the conduction electrons at any Bloch state. As a consequence, any cumulant average in the perturbative expansion that contains localized electrons at different sites or conduction electrons in different states, or both, is automatically zero: this is the basic reason for the validity of the linked cluster expansion in the PAM.

When the localization is very strong, a usual approximation is to consider a PAM with an infinite repulsion $U$ between the two localized electrons at the same site, and one can then neglect the double occupation. This model was discussed by Hewson, ${ }^{12}$ who applied Hubbard's results ${ }^{2}$ to the PAM without an explicit derivation. He calculated the lowest order correction to the magnetic susceptibility of the PAM, as well as the one-electron's GF in the "chain approximation" (CHA). In his Ph.D. thesis Martine ${ }^{13}$ presented an explicit derivation of the linked cluster theorem for the PAM's free energy, and calculated the GF both in the chain and in the "multiple loops approximation" (MLA) which shall be discussed in a following paper. There we shall report the application of the CHA and the MLA to a rectangular conduction band and to the "atomic" model (band with zero width). In the "atomic" case it is possible to compare the results of the two approximations with the exact results, so that a better understanding of their validity can be obtained.

In the derivation of the perturbative expansion, Hubbard $^{2}$ employed "external fields" $\xi$ that appear in the generating function of the cumulants of the problem and are Grassmann variables, ${ }^{14}$ but he only presented diagrammatic rules for zero "external fields." We have extended the diagrammatic expansion to $\xi \neq 0$, and the results are presented in Sec. III together with the $\xi=0$ case, because this only requires minimum changes to the diagrammatic rules. We shall use the $\xi \neq 0$ case in a future work.

\section{THE PAM HAMILTONIAN WITH HUBBARD OPERATORS}

In this section we shall present a generalization of the PAM to a periodic array of ions with a rather general scheme of localized levels. We shall first write the Hamiltonian of the PAM with the usual fermion operators, showing how to express it employing the less familiar Hubbard $X$ operators, and as a second step we shall introduce the more general model.

The Hamiltonian for the PAM is

$$
\begin{aligned}
H= & \sum_{\vec{k}, \sigma} E_{\vec{k}, \sigma} C_{\vec{k}, \sigma}^{\dagger} C_{\vec{k}, \sigma}+\sum_{j, \sigma} E_{\sigma} f_{j, \sigma}^{\dagger} f_{j, \sigma} \\
& +U \sum_{j} n_{j, \sigma} n_{j, \bar{\sigma}}+H_{h},
\end{aligned}
$$

where the operators $C_{\vec{k}, \sigma}^{\dagger}$ and $C_{\vec{k}, \sigma}$ are the creation and destruction operators of conduction band electrons ( $c$ electrons) with wave vector $\vec{k}$, component of spin $\sigma$ and energies $E_{\vec{k}, \sigma}$. The $f_{i, \sigma}^{\dagger}$ and $f_{i, \sigma}$ are the corresponding operators for the $f$ electrons in the Wannier localized state at site $j$, with spin component $\sigma$ and energy $E_{\sigma}$ that is site independent. The third term is the Coulomb repulsion between the localized electrons at each site where $n_{j, \sigma}=f_{j, \sigma}^{\dagger} f_{j, \sigma}$ is the number of $f$ electrons with spin component $\sigma$ at site $j$ and the symbol $\bar{\sigma}$ denotes the spin component opposite to $\sigma$. The fourth term $H_{h}$ describes the hybridization between the localized and conduction electrons:

$$
H_{h}=\sum_{j, \vec{k}, \sigma}\left(V_{j, \vec{k}, \sigma} f_{j, \sigma}^{\dagger} C_{\vec{k}, \sigma}+V_{j, \vec{k}, \sigma}^{*} C_{\vec{k}, \sigma}^{\dagger} f_{j, \sigma}\right),
$$

with a coupling strength given by

$$
V_{j, \vec{k}, \sigma}=\frac{1}{\sqrt{N_{s}}} V_{\sigma}(\vec{k}) \exp \left(i \vec{k} \cdot \vec{R}_{j}\right)
$$

where $V_{\sigma}(\vec{k})$ is independent of the wave vector $\vec{k}$ when the mixing is purely local and $N_{s}$ is the number of sites in the system.

If we consider that the local repulsion between $f$ electrons is infinite $(U \rightarrow \infty)$, so that the double occupancy at any site is zero, we can make the term in the Hamiltonian proportional to $U$ disappear by employing Hubbard operators. To this purpose we consider first the definition of the $X$ operators: the $X_{j, b a}$ transforms the state $|a\rangle$ at site $j$ into the state $|b\rangle$ at the same site, and we assume that $|a\rangle$ and $|b\rangle$ are eigenstates of the number of electrons. We say that $X_{j, b a}$ is of the Fermi type when $|a\rangle$ and $|b\rangle$ differ by an odd number of fermions, and that it is of the Bose type when they differ by an even number of electrons. By definition, two $X$ operators of the Fermi type at different sites anticommute, and commute when at least one of them is of the Bose type. The algebra of these operators when they are at the same site is defined by their product rule

$$
X_{j, a b} X_{j, c d}=\delta_{b, c} X_{j, a d}
$$


and they are neither fermions nor bosons. For infinite $U$, the only $f$-electron states at any site $j$ are the vacuum $|j, 0\rangle$ and the two states $|j, \sigma\rangle$ that have one electron with spin component $\sigma$, and the only Fermi-type operators that we shall need in this case are $X_{j, o \sigma}$ and its Hermitian conjugate $X_{j, \sigma o}=X_{j, o \sigma}^{\dagger}$. Projecting $H$ into the subspace without doubly occupied $f$-electron states we obtain the PAM Hamiltonian for infinite $U$ :

$$
\begin{aligned}
H= & \sum_{\vec{k}, \sigma} E_{\vec{k}, \sigma} C_{\vec{k}, \sigma}^{\dagger} C_{\vec{k}, \sigma}+\sum_{j \sigma} E_{j \sigma} X_{j, \sigma \sigma} \\
& +\sum_{j, \vec{k}, \sigma}\left(V_{j, \vec{k}, \sigma} X_{j, o \sigma}^{\dagger} C_{\vec{k} \sigma}+V_{j, \vec{k}, \sigma}^{*} C_{\vec{k} \sigma}^{\dagger} X_{j, o, \sigma}\right)
\end{aligned}
$$

where $X_{j, \sigma \sigma}=X_{j, o \sigma}^{\dagger} X_{j, o \sigma}$ is the projector into the state $|j, \sigma\rangle$. The "completeness" relation

$$
X_{j, o o}+X_{j, \sigma \sigma}+X_{j, \overline{\sigma \sigma}}=I
$$

gives the conservation of probability in the space of the localized states at site $j$.

The generalization of Eq. (2.5) to the case of several configurations with a rather arbitrary choice of states is

$$
H=\sum_{\vec{k}, \sigma} E_{\vec{k}, \sigma} C_{\vec{k}, \sigma}^{\dagger} C_{\vec{k}, \sigma}+\sum_{j a} E_{j a} X_{j, a a}+H_{h}
$$

where

$$
H_{h}=\sum_{j b a, \vec{k} \sigma}\left(V_{j b a, \vec{k} \sigma} X_{j, b a}^{\dagger} C_{\vec{k} \sigma}+V_{j b a, \vec{k} \sigma}^{*} C_{\vec{k} \sigma}^{\dagger} X_{j, b a}\right) .
$$

The $a$ and $b$ summations are over all the states $|a\rangle$ and $|b\rangle$ that we want to include in the model, and the only restriction is that any hybridization constant must vanish unless state $|a\rangle$ has just one electron more than the state $|b\rangle$ : this last condition is necessary to satisfy the conservation of electrons. In this general case, the energies $E_{j, a}$ include all the Coulomb repulsions of the type described by the third term in Eq. (2.1); in Sec. III we shall derive expressions valid for Eq. (2.7), but most of the examples in the following paper correspond to the PAM for infinite $U$, described by Eq. ( 2.5).

As we are interested in the grand canonical ensemble of electrons, we should replace the total Hamiltonian $H$ by

$$
\mathcal{H}=H-\mu\left\{\sum_{\vec{k}, \sigma} C_{\vec{k}, \sigma}^{\dagger} C_{\vec{k}, \sigma}+\sum_{j a} \nu_{a} X_{j, a a}\right\},
$$

where $X_{j, a a}$ is the occupation number operator of state $|a\rangle$ at site $j$, and $\nu_{a}$ is the number of electrons in that state. This transformation is easily performed by changing the energies $E_{j, a}$ of all ionic states $|a\rangle$ into

$$
\epsilon_{j, a}=E_{j, a}-\mu \nu_{a}
$$

and the energies $E_{\vec{k}, \sigma}$ of the conduction electrons into

$$
\epsilon_{\vec{k} \sigma}=E_{\vec{k} \sigma}-\mu \text {. }
$$

One should remember that in this formalism, the Fourier transforms of the one-electron GF for real times have their frequencies displaced by the chemical potential $\mu$. As usual, we shall split Eq. (2.9) into an unperturbed part $\mathcal{H}_{0}$ and a perturbation $H_{h}$ :

$$
\mathcal{H}=\mathcal{H}_{0}+H_{h}
$$

\section{THE PERTURBATION EXPANSION}

To introduce the cumulants employed in the diagrammatic expansion, it is necessary to add the following "external field" term to the Haniltonian:

$$
H_{e}(\xi)=-\sum_{\gamma} \xi_{\gamma}(\tau) Y_{\gamma}=H_{e}[\xi(\tau)]
$$

where the $Y_{\gamma}$ describe all the operators $X_{j, b a}$ and $C_{\vec{k} \sigma}$ that appear in Eq. (2.7), as well as their Hermitian conjugates, and the subindex $\gamma$ should carry all the necessary identification. The "external fields" $\xi_{\gamma}(\tau)$ are Grassmann variables, ${ }^{14}$ which anticomnute between themselves and with all the $Y_{\gamma}$ of Eq. (3.1): this is an essential property for the derivation of the diagramnatic expansion.

\section{A. Linked cluster theorem for the grand canonical potential}

First we consider the grand partition function (GPF) in the presence of the Grassmann fields $\xi$

$$
\mathcal{Z}(\beta, \xi)=\operatorname{Tr}\{U(\beta, \xi)\},
$$

where $\beta=1 / k T$ and $U(\beta, \xi)$ is a natural extension of the evolution operator to imaginary times and nonzero $\xi$ (cf. Appendix A). To nuake an expansion of $\mathcal{Z}(\beta, \xi)$ employing $H_{h}$ as perturbation we use [cf. Eqs. (A9) and (A16)]

$$
U(\beta, \xi)=U(\beta, 0, \xi)=\exp \left(-\beta \mathcal{H}_{0}\right) \mathcal{S}_{\mathcal{H}_{0}}(\beta, \xi)
$$

where

$$
\mathcal{S}_{\mathcal{H}_{0}}(\beta, \xi)=\exp +\left\{-\int_{0}^{\beta} d \tau\left[H_{h}(\tau)+H_{e}(\tau, \xi)\right]\right\} .
$$

The symbol exp + means ${ }^{11}$ that we order all the terms in the formal expansion of the exponential with the variable $\tau$ increasing to the left, and that there is a change of sign in any term of the expansion when two operators of the Fermi type must be exchanged to put them in the correct ordering. In what follows we shall understand this type of ordering for any expression inside parentheses with a subindex + .

Following the standard procedure, we make a formal expansion of this operator, taking advantage of the fact that $H_{h}(\tau)$ and $H_{e}(\tau, \xi)$ are of the Bose type, and can be moved without changes of sign inside the time ordered products. 
There are two possible choices for this expansion: either expand the whole exponential in Eq. (3.4) or expand only $\mathcal{S}_{\mathcal{H}_{0}}^{0}(\beta)$ employing the relation [cf. Eq. (A17)]

$$
\mathcal{S}_{\mathcal{H}_{0}}(\beta, \xi)=\left[\mathcal{S}_{\mathcal{H}_{0}}^{0}(\beta) \mathcal{E}(\beta, \xi)\right]_{+},
$$

where for $V=H_{h}$ we have [cf. Eqs. (A18) and (A19)]

$$
\mathcal{S}_{\mathcal{H}_{0}}^{0}(\beta)=\exp +\left\{-\int_{0}^{\beta} d \tau H_{h}(\tau)\right\}
$$

and

$$
\mathcal{E}(\beta, \xi)=\exp +\left\{-\int_{0}^{\beta} d \tau H_{e}(\tau, \xi)\right\} .
$$

The operators $H_{h}(\tau)$ and $H_{e}(\tau, \xi)$ are $H_{h}$ and $H_{e}(\xi)$ in the interaction picture, respectively, and the two choices of expansion give different diagram rules, which coincide in the $\xi=0$ limit. We choose to expand $\mathcal{S}_{H_{0}}^{0}(\beta)$ in Eq. (3.6) because the diagrammatic rules are then essentially the same derived for $\xi=0$, except for the rule derived from the conservation of particles, which fails when $\xi \neq 0$. A typical term in the expansion of $\mathcal{Z}(\beta, \xi)$ is

$$
\begin{aligned}
\frac{(-1)^{n}}{n !} \int_{0}^{\beta} d \tau_{1} \cdots \int_{0}^{\beta} d \tau_{n} \operatorname{Tr}\left\{\exp \left(-\beta H_{0}\right)\right. \\
\left.\times\left[H_{h}\left(\tau_{1}\right) \cdots H_{h}\left(\tau_{n}\right) \mathcal{E}(\beta, \xi)\right]_{+}\right\},
\end{aligned}
$$

which is the usual result but for the presence of $\mathcal{E}(\beta, \xi)$, and this last is the identity when $\xi=0$. We denote with $\mathcal{Z}_{0}(\beta, \xi)$ the first term in the expansion, which is equal to the unperturbed $\mathcal{Z}(\beta, \xi)$ (i.e., with $H_{h}=0$ ).

To proceed, we substitute $H_{h}(\tau)$ in Eq. (3.8) by Eq. (2.8) in the interaction picture, which we write in the more compact form

$$
H_{h}(\tau)=\sum_{l, l^{\prime}} V\left(l, l^{\prime}\right) Y(l) Y\left(l^{\prime}\right)
$$

where

$$
Y(l) \equiv Y_{\gamma}(\tau)=\exp \left(\tau \mathcal{H}_{0}\right) Y_{\gamma} \exp \left(-\tau \mathcal{H}_{0}\right)
$$

is the operator $Y_{\gamma}$ in the interaction picture. The only nonzero coupling coefficients $V\left(l, l^{\prime}\right)$ are those that correspond to the correct combination of indices $l$ and $l^{\prime}$ in Eq. (2.7) and a factor $1 / 2$ is not necessary in Eq. (3.9) if we choose to retain only terms in which $Y(l)$ corresponds to the $f$ electrons and $Y\left(l^{\prime}\right)$ to the conduction electron [to achieve this ordering in the second term of the parentheses in Eq. (2.8) one must anticommute two Fermi-type operators]. By substitution in Eq. (3.8) we find

$$
\begin{aligned}
& \frac{(-1)^{n}}{n !} \mathcal{Z}_{0}(\beta, \xi) \int_{0}^{\beta} d \tau_{1} \sum_{l_{1}, l_{1}^{\prime}} V\left(l_{1}, l_{1}^{\prime}\right) \cdots \int_{0}^{\beta} d \tau_{n} \\
& \quad \times \sum_{l_{n}, l_{n}^{\prime}} V\left(l_{n}, l_{n}^{\prime}\right)\left\langle\left[Y\left(l_{1}\right) Y\left(l_{1}^{\prime}\right) \cdots Y\left(l_{n}\right) Y\left(l_{n}^{\prime}\right)\right]_{+}\right\rangle^{\xi},
\end{aligned}
$$

where we employ the " $\xi$ average" defined by

$$
\begin{array}{r}
\left\langle\left[Y\left(l_{1}\right) Y\left(l_{1}^{\prime}\right) \cdots Y\left(l_{n}\right) Y\left(l_{n}^{\prime}\right)\right]_{+}\right\rangle^{\xi} \\
\equiv \frac{\left\langle\left[Y\left(l_{1}\right) Y\left(l_{1}^{\prime}\right) \cdots Y\left(l_{n}\right) Y\left(l_{n}^{\prime}\right) \mathcal{E}(\beta, \xi)\right]_{+}\right\rangle}{\langle\mathcal{E}(\beta, \xi)\rangle}
\end{array}
$$

and $\langle\ldots\rangle$ is here the usual unperturbed average when $H_{h}$ and $\xi$ are taken null (cf. Appendix A), so that $\mathcal{Z}_{0}(\beta, \xi)=$ $\langle\mathcal{E}(\beta, \xi)\rangle \operatorname{Tr}\left\{\exp \left(-\beta \mathcal{H}_{0}\right)\right\}$. The expansion in Eq. (3.11) looks the same as the one with $\xi=0$ except that we have " $\xi$ averages" instead of common averages. From this point onwards, the derivation of the cumulant expansion proceeds through a rather general property of cumulants, which reads as follows in the present case (cf. Ref. 3, p. 131).

Theorem 3.1. " $\left\langle\left[Y\left(l_{1}\right) Y\left(l_{2}\right) \cdots Y\left(l_{n}\right)\right]_{+}\right\rangle^{\xi}$ is equal to the sum of products of cumulant correlations in which each term corresponds to a partition of the arguments $l_{1} l_{2} \cdots l_{n}$ and every possible partition appears once and only once." Every term has the sign of the permutation that takes the ordering of all their Fermi-type operators into the ordering that the same operators have at the $\xi$ average.

The sentence in italics has been added to the verbatim statement in Ref. 3, and it is necessary here because all the $Y\left(l_{j}\right)$ are Fermi-type operators. Theorem 3.1 has been phrased so that it is also valid when some of the $Y(j)$ operators are of the Bose type: this more general type of average was considered also in Ref. 2 , and can be employed to study properties like the magnetic susceptibility.

In Appendix B we discuss the basic formula that leads to theorem 3.1, and we emphasize that the $\xi$ averages are not numbers but belong to the Grassmann space and do not necessarily commute between themselves, e.g.,

$$
\left\langle\left[Y\left(l_{1}\right)\right]_{+}\right\rangle^{\xi}\left\langle\left[Y\left(l_{2}\right)\right]_{+}\right\rangle^{\xi}=-\left\langle\left[Y\left(l_{2}\right)\right]_{+}\right\rangle^{\xi}\left\langle\left[Y\left(l_{1}\right)\right]_{+}\right\rangle^{\xi} .
$$

Theorem 3.1 is valid for nonzero $\xi$ and $H_{h}$, but the $\xi$ cumulants that appear from the decomposition of the $\xi$ averages in Eq. ( 3.11 ) correspond to those defined in Eq. (A28) but with $H_{h}=0$, i.e.,

$$
\left\langle\left[Y\left(l_{1}\right) \cdots Y\left(l_{n}\right)\right]_{+}\right\rangle_{c}^{\xi}=\delta_{1} \delta_{2} \cdots \delta_{n} \ln \left[\mathcal{Z}_{0}(\beta, \xi)\right],
$$

where we have used the notation $\delta_{j}$ for the functional derivative: $\left[\delta / \delta \xi\left(l_{j}\right)\right]$. We shall also use $Y(j) \equiv Y\left(l_{j}\right)$ and $V(i, j) \equiv V\left(l_{i}, l_{j}\right)$ in places where this abbreviation would not lead to a possible confusion. Two typical examples of theorem 3.1 are

$$
\begin{aligned}
\left\langle(1,2)_{+}\right\rangle^{\xi} & =\left\langle(1,2)_{+}\right\rangle_{c}^{\xi}+\left\langle(1)_{+}\right\rangle_{c}^{\xi}\left\langle(2)_{+}\right\rangle_{c}^{\xi}, \\
\left\langle(1,2,3)_{+}\right\rangle^{\xi}= & \left\langle(1,2,3)_{+}\right\rangle_{c}^{\xi}+\left\langle(1)_{+}\right\rangle_{c}^{\xi}\left\langle(2,3)_{+}\right\rangle_{c}^{\xi} \\
& -\left\langle(2)_{+}\right\rangle_{c}^{\xi}\left\langle(1,3)_{+}\right\rangle_{c}^{\xi}+\left\langle(3)_{+}\right\rangle_{c}^{\xi}\left\langle(1,2)_{+}\right\rangle_{c}^{\xi} \\
& +\left\langle(1)_{+}\right\rangle_{c}^{\xi}\left\langle(2)_{+}\right\rangle_{c}^{\xi}\left\langle(3)_{+}\right\rangle_{c}^{\xi},
\end{aligned}
$$


where we have further abbreviated $\left\langle\left[Y\left(l_{1}\right) Y\left(l_{2}\right) \cdots Y\left(l_{n}\right)\right]_{+}\right\rangle^{\xi}=\left\langle(1,2 \cdots n)_{+}\right\rangle^{\xi}$.

The next step in the derivation is to apply the general property stated above to the " $\xi$ averages" in Eq. (3.11). As in this case $H_{h}=0$, any two $Y$ operators that correspond to $f$ electrons at different sites, or to conduction electrons with different $\vec{k}$ or $\sigma$, or to one $f$ electron and a conduction electron, are statistically independent even when $\xi \neq 0$. As a consequence, the only cumulants that appear in Eq. (3.11) contain only $Y$ operators of $f$ electrons at the same site or only $C$ operators with the same $\vec{k}$ or $\sigma$, and all other cumulants in the expansion vanish. The graphic expansion that follows is based in this result. It is then clear that the key property is not quite the locality of the $Y$ but rather their stochastic independence. This fact permits the same type of cumulant expansion employed for the Hubbard model, ${ }^{2}$ where all the operators that correspond to our $Y$ are "local" operators, while in the PAM we also have cumulants of $C$ operators that correspond to "extended" Bloch functions. The property just discussed is the basis for an expansion employing graphs, ${ }^{3}$ i.e., a set of vertices (points) and edges (lines). We shall assign cumulants to the vertices, and represent with a filled circle any " $f$-electron vertex" (FV) of cumulants of $X$ operators, and with an open circle any "conduction vertex" (CV) of cumulants of $C_{\vec{k} \sigma}^{\dagger}$ and $C_{\vec{k} \sigma}$ operators. As discussed above, the only nonzero cumulants correspond either to $X$ operators, all at the same site, or to $C$ operators, all with the same $\vec{k}$ and $\sigma$. In the expansion of the GPF all the vertices are "internal," i.e., all their $Y(l)$ operators originate from the perturbation $H_{h}$ and appear summed over all the indices $\gamma$ and integrated over $\tau$, both characterized by the argument $l$ in the $Y(l)$. These graphs with only internal lines are called "vacuum graphs" 2 (cf. a few examples of graphs and vertices in Fig. 1).

Each internal edge is incident at two distinct vertices, and because of the interaction employed, it can only join a CV with an FV. The edges are associated to the coefficients in $H_{h}$ [e.g., the $V\left(l, l^{\prime}\right)$ in Eq. (3.9)].

After decomposing the $\xi$-averages of Eq. (3.11) into cumulants, we associate a collection of graphs to each order $n$ in the expansion, and we give below the rules to construct all the necessary graphs. There are both connected and disconnected graphs ${ }^{3}$ in these collections, and in the next section we give detailed rules to calculate the contribution associated to any graph. Except for some counting details, the contribution of a disconnected graph is equal to the product of the individual contributions of the connected graphs that constitute the whole graph; this property is valid because in the calculation of the graph contribution there are no excluded site restrictions. ${ }^{7}$ Notice that although the contributions of graphs are Grassmann numbers for $\xi \neq 0$, all the vacuum graphs have an even number of $Y$ operators [because of the form of the interaction Eq. (2.8)] and their contributions commute with any other graph contribution. The rest of the GPF calculation then follows closely the derivation for the classical Ising model in Ref. 3.

It is convenient to label with an index $\alpha$ each of the

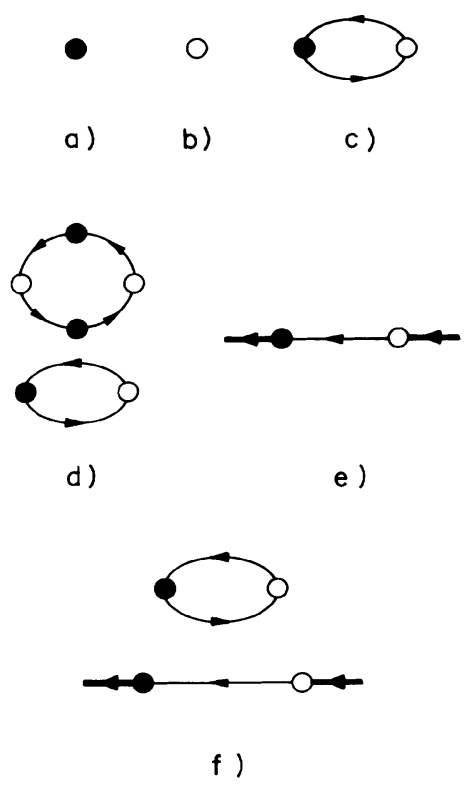

FIG. 1. (a) An $f$-electron vertex (FV). (b) A conduction electron vertex (CV). (c) A connected vacuum graph (order $n=2)$. (d) A disconnected graph $(n=6)$. (e) A connected rooted graph $(n=1)$. (f) A disconnected rooted graph $(n=3)$.

topologically distinct connected graphs, and denote with $W_{\alpha}$ the contribution of graph $\alpha$. Adding up all the terms like Eq. (3.11) one obtains

$$
\mathcal{Z}(\beta, \xi)=\mathcal{Z}_{0}(\beta, \xi) \exp \left\{\sum_{\alpha} W_{\alpha}\right\},
$$

and the grand canonical potential (GCP) for nonzero hybridization is then

$$
\Omega(\beta, \xi)=-T \ln \{\mathcal{Z}(\beta, \xi)\}
$$

(in the whole paper we shall measure $T$ in units of the Boltzmann constant $k_{B}$ ).

The change in the GCP due to $H_{h}$ is then

$$
\Omega(\beta, \xi)-\Omega_{0}(\beta, \xi)=-T\left\{\sum_{\alpha} W_{\alpha}\right\},
$$

where $\Omega_{0}(\beta, \xi)$ is the value of $\Omega(\beta, \xi)$ when $H_{h}=0$. The corresponding equation for the Hubbard model (with a correction due to the mean-field treatment) is given in Ref. 2 [cf. Eq. (35)]. In Eq. (3.17) we follow Hubbard's notation, so that our $W_{\alpha}$ is already divided by a symmetry factor $g_{\alpha}$, as discussed in the next section. For this reason, it is $W_{\alpha}$ that appears, rather than $W_{\alpha} / g_{\alpha}$ as happens in Ref. 3.

From these two equations we get the following.

Theorem 3.2 (The "linked cluster theorem" for the $G C P)$. The change in the GCP due to the perturbation is equal to the sum of the contributions of all the topologically distinct connected vacuum graphs.

The concept of "topologically distinct" graphs seems 
clear, but in Ref. 3 (p. 125) there is an explicit definition. To construct any vacuum graph of order $n$ for $\xi \neq 0$ we follow rule 3.1 .

Rule 3.1.

(1) Mark any number of FV (filled circles) and of CV (open circles).

(2) Add $n$ internal lines (edges), each one connecting one FV to a $\mathrm{CV}$.

(3) At least one line must run to each vertex, so that the maximum number of each type of vertices is equal to the order $n$ of the term [i.e., Eq. (3.8)] in the perturbation sum.

(4) Associate in pairs all the internal lines running to each vertex in some arbitrary fashion (when there is an odd number of lines one of them would not be paired). In this way the lines form open and closed loops: assign to each of these loops a definite sense, the sense being indicated by arrows on the lines.

Note that there are no restrictions to the number of lines that reach any vertex when $\xi \neq 0$ (cf. Fig. 2). When $\xi=0$ the $\xi$ averages satisfy particle conservation, and because of the form of $H_{h}$ [the only internal lines in the PAM correspond to one-electron creation and destruction operators (cf. Eq. (3.9)] only an even number of edges can reach any vertex.

For $\xi=0$, Wick's theorem, together with the general property of the averages discussed above, shows that cumulants with more than two unperturbed Fermi operators (the $C_{\vec{k} \sigma}^{\dagger}$ and $C_{\vec{k} \sigma}$ in the present case) must vanish. We summarize the results for $\xi=0$ in the following rule.

Rule 3.2. For $\xi=0$ employ rule 3.1 with the following restriction.

(5) Only an even number of internal lines can reach any vertex in a vacuum graph. There can only be two lines running to each $\mathrm{CV}$.

The cumulants at any vertex with an odd number of lines vanish at the final step of the calculation when the physical quantities are obtained by taking $\xi=0$. Nevertheless, the GF of interest can be expressed as derivatives of $\mathcal{Z}(\beta, \xi)$ with respect to $\xi$ [cf. Eq. (A26)], and a CV with only one internal line can be transformed by this procedure into another with two lines that would not vanish when $\xi=0$. As the contribution of any $\mathrm{CV}$ with three or more lines would vanish when $\xi=0$ because of Wick's theorem, we shall then consider a CV with only one or two edges when $\xi \neq 0$ : in Fig. 2 there are several

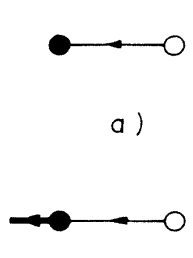

c)

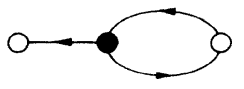

b)

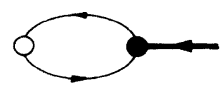

d )
FIG. 2. Several graphs with an odd number of edges running to one or more vertices. Their contributions vanish when $\xi=0$. (a) and (b) Vacuum graphs. (c) and (d) Rooted graphs: thick lines correspond to external edges. typical graphs that contribute for $\xi \neq 0$ but vanish for $\xi=0$.

To apply the linked cluster theorem, we must first construct all the topologically distinct graphs following rules 3.1 or 3.2 , and then sum their corresponding contributions according to rule 3.5 in Sec. III B.

\section{B. Diagrammatic rules in real space and imaginary time}

In the present section we consider not only vacuum graphs, but also "rooted graphs" (i.e., graphs with external lines), which appear in the calculation of the averages $\left\langle[\hat{Y}(1) \cdots \hat{Y}(r)]_{+}\right\rangle^{V \xi}$ [cf. Eq. (A24)], i.e., the Green's functions discussed in the next section. In the perturbation expansion one should then calculate $\xi$ averages which contain several fixed $Y$ operators, besides those that arise from the $n$ factors $H_{h}(\tau)$ of the $n$ th-order term. To represent an external operator in a graph, we shall employ a thick segment attached to a vertex of the same $f$ or $c$ character, and a few rooted graphs are shown in Figs. 1 and 2. In the interaction $H_{h}$ there are only operators $Y_{\gamma}$ that create or destroy a single electron, but one could also consider external Hubbard operators that are more general, as is done in Ref. 2. We think that it would not be useful to make that extension here in a very general way, but without enlarging too much the formulation, we can include external Hubbard operators of the Bose type that change the state of a local site without changing their number. Those operators are useful to calculate properties like susceptibility, and we shall consider them here as possible external operators. In what follows, Fermi-type operators would refer to those that create or destroy one electron, and Bose-type to those that do not change the number of electrons. We shall call Fermi lines or Bose lines those that represent the corresponding external operators in a graph, and all the internal lines in the present model are of the Fermi type. The rule for drawing graphs of $n$th order in perturbation theory, when $\xi \neq 0$ and there are $r$ fixed operators $Y(1) \cdots Y(r)$ that can be of Fermi-type or of Bose-type, is then the following.

Rule 3.3.

(1) Mark any number of FV and of CV.

(2) Draw $r$ external lines, labeled to correspond to the $Y(1) \cdots Y(r)$ external operators. Each external line runs to an FV when the $Y$ operator is associated to $f$ electrons, and to a $\mathrm{CV}$ when it is associated to a conduction electron. Draw $n$ internal lines, each one connecting one FV to a CV.

(3) At least one line, internal or external, runs to each vertex.

(4) Associate in pairs all the internal lines and Fermi external lines running to each vertex in some arbitrary fashion (when there is an odd number of lines one of them would not be paired). In this way the Fermi lines form open and closed loops. Assign to each of these loops a definite sense, the sense being indicated by arrows on the lines.

When $\xi=0$ we have the following. 
Rule 3.4. Employ rule 3.3 with the following restriction.

(5) The total number of Fermi lines, internal or external, running to each vertex, is even. There can only be two Fermi lines for each CV.

To give the contribution of the diagrams discussed above, we shall first consider the general Hamiltonian defined by Eq. (2.7), discussing later the contribution for the PAM with infinite $U$ as a special case. The rules are essentially the same stated by Hubbard in Ref. 2 , but we present here their adaptation to our model. At this point it is necessary to be more specific about the argument $l$ of the operators $Y_{l}$ in Eq. (3.9). When the corresponding $Y_{\gamma}$ is a Fermi-type $X_{j, b a}$, we use $\gamma=(f ; j, \alpha, u)$, with $u=-$, and the single index $\alpha$ identifies the transition $|a\rangle \rightarrow|b\rangle$, with the same restriction stated after Eq. (2.8), namely that state $|a\rangle$ has just one electron more than the state $|b\rangle$. The inverse transition (operator $X_{j, b a}^{\dagger}$ ) is described by the same $\alpha$ but with $u=+$. The $j$ identifies the site, the imaginary time in the interaction picture [cf. Eq. (3.10)] is $\tau$, and $f$ is only used when necessary to avoid confusion. When $Y_{\gamma}$ is $C_{\vec{k} \sigma}$ we use $\gamma=(c ; \vec{k}, \sigma, u)$ with $u=-$ and change to $u=+$ for $C_{\vec{k} \sigma}^{\dagger}$. It is not necessary to assign a $u$ parameter to the Bose-type operators, but to unify the notation we shall keep the $u$ and set always $u=1$ for these operators. The only restriction on the two states $|a\rangle$ and $|b\rangle$ of the transition $\alpha=(b, a)$ for Bose-type operators is that they have the same number of electrons.

To avoid repeating the same term in Eq. (3.9) we assumed that $V\left(l, l^{\prime}\right)$ is nonzero only when the first index correspond to an $X$ operator. These coefficients do not depend on $\tau$ or $\tau^{\prime}$, and comparing with Eq. (2.8) it is convenient to introduce $v(j, \alpha, \vec{k}, \sigma, u)$ :

$$
\begin{aligned}
& v(j, \alpha, \vec{k}, \sigma,+)=V(f ; j, \alpha,+; c ; \vec{k}, \sigma,-)=V_{j, b a, \vec{k}, \sigma} \\
& v(j, \alpha, \vec{k}, \sigma,-)=-V(f ; j, \alpha,-; c ; \vec{k}, \sigma,+)=V_{j, b a, \vec{k}, \sigma^{*}}^{*}
\end{aligned}
$$

The minus sign that should appear with $V_{j, b a, \vec{k}, \sigma}^{*}$ because we anticommuted two Fermi-type operators from Eq. (2.8) will be absorbed in the rules for the sign of the graph contributions when $\xi=0$ (cf. Appendix C).

Rule 3.5 (contribution of a given graph with $\xi \neq 0$ ). To calculate the contribution of a diagram obtained from rules 3.3 or 3.4 , do the following.

(1) Assign to each FV a site label $j_{s}$. To each internal line assign an imaginary time $\tau_{s}$ and an index $u_{s}$. To the FV side of the internal line assign to the $X$ operator the dunimy labels $\alpha_{s}$ and $\pm u_{s}$ and to the CV side assign to the $C$ operator the dummy labels $\vec{k}_{s}, \sigma_{s}$, and $\mp u_{s}$. Use $+u_{s}$ at the side of the edge to which points the arrow of item (4) of rules $3.1-3.4$ and $-u_{s}$ at the opposite side. Each external line is already associated by its label to a $Y$ operator (cf. item 2) in rule 3.3. Note that the external operators could also be of the Bose type.

(2) Form the product of the following factors.

(a) For each FV a factor

$$
\left\langle\left[Y\left(f ; j_{s}, \alpha_{1}, \pm u_{1}, \tau_{1}\right) \cdots Y\left(f ; j_{s}, \alpha_{p}, \pm u_{p}, \tau_{p}\right)\right]_{+}\right\rangle_{c}^{\xi}
$$

when there are $p$ lines running to that vertex. The $\alpha, u$, and $\tau$ are equal to their assigned values for the external lines.

(b) For each CV a factor

$$
\left\langle\left[Y\left(c ; \vec{k}_{s}, \sigma_{1}, \mp u_{1}, \tau_{1}\right) \cdots Y\left(c ; \vec{k}_{s}, \sigma_{p}, \mp u_{p}, \tau_{p}\right)\right]_{+}\right\rangle_{c}^{\xi},
$$

when there are $p$ lines running to that vertex $(p=1,2)$. The $u, \sigma$, and $\tau$ are equal to their assigned values for the external lines.

(c) A factor $v(j, \alpha, \vec{k}, \sigma, \pm u)$ for each internal line joining an FV at site $j$ with labels $\alpha, \pm u$ to a CV with labels $\vec{k}, \sigma$, and $\mp u$. When $\xi \neq 0$, multiply also into the sign $\pm u$ employed in this factor (this $\pm u$ is absorbed in the sign rules for $\xi=0$ ).

(d) A $\delta\left(j_{s}, j_{i}\right)$ for each external line $X$ operator at site $j_{i}$ running to an FV site labeled with $j_{s}$, and a $\delta\left(\vec{k}_{s}, \vec{k}_{i}\right)$ for each external line $C$ operator with wave vector $\vec{k}_{i}$ running to a CV with an internal line labeled with $\vec{k}_{s}$. [The labels $j_{s}$ and $\vec{k}_{s}$ are dummy labels, but the Kronecker deltas in the present item take care of fixing their values when there is an external line running to an FV or to a CV. When $\mathcal{H}_{0}$ is independent of spin, the $\xi$-cumulant of (b) vanishes unless all the $\sigma$ are equal.]

(e) A factor \pm 1 determined by the rules given in Appendix $\mathrm{C}$.

(f) A factor $1 / g$ determined by the rules given in Appendix $D$.

(3) Sum the resulting product with respect to the following.

(a) The site labels $j_{s}$ of all the FV and the $\alpha_{s}$ labels of the internal $X$ operator of all the FV.

(b) The labels $\vec{k}_{s}$ of all the CV and the $\sigma_{s}$ labels of the internal $C$ operators of all the CV.

(c) The labels $u_{s}$ of all the internal lines.

(d) Integrate between 0 and $\beta$ over the imaginary times $\tau_{s}$ associated to all the internal lines.

When $\xi=0$ the number of topologically distinct diagrams is reduced very much but only a few modifications to rule 3.5 are necessary, and we have the following.

Rule 3.6 (contribution of a given diagram with $\xi=0$ ). Follow rule 3.5, changing item 2(b) into the following. $2\left(b^{\prime}\right)$ For each CV put a factor

$$
\left\langle\left[Y\left(c ; \vec{k}_{s}, \sigma_{2},-u_{2}, \tau_{2}\right) Y\left(c ; \vec{k}_{s}, \sigma_{1},+u_{1}, \tau_{1}\right)\right]_{+}\right\rangle_{c}^{\xi},
$$

where the labels with 1 correspond to the edge with the arrow pointing towards the $\mathrm{CV}$.

This change is convenient to simplify the calculation of the sign in item " $2(\mathrm{e})$ of rule 3.5 ," as discussed in Appendix C. 


\section{Diagrammatic expansion of the Green's functions}

We define the Green's functions in the presence of external fields $\xi$ as the averages [cf. Eq. (A24)]

$$
G(1,2, \ldots, r ; \xi)=\left\langle[\hat{Y}(1) \cdots \hat{Y}(r)]_{+}\right\rangle^{V \xi}
$$

where we have replaced the set of parameters $l_{s}=\left(\gamma_{s}, \tau_{s}\right)$ that describe the operators $\hat{Y}\left(l_{s}\right)$ with the indices $s$ [we use $\gamma=(f ; j, \alpha, u)$ or $\gamma=(c ; \vec{k}, \sigma, u)]$, and we shall follow this notation when it would not lead to confusion. From Eq. (A27) we obtain

$$
G(1,2, \ldots, r ; \xi)=\frac{\left\langle\left[Y(1) \cdots Y(r) \mathcal{S}_{\mathcal{H}_{0}}^{0}(\beta) \mathcal{E}(\beta, \xi)\right]_{+}\right\rangle}{\left\langle\left[\mathcal{S}_{\mathcal{H}_{0}}^{0}(\beta) \mathcal{E}(\beta, \xi)\right]_{+}\right\rangle}
$$

and we can then use the same type of expansion employed in the linked cluster theorem in Sec. III A, both for the numerator and the denominator of Eq. (3.25). The numerator is equal to the sum of the contribution of all the topologically distinct graphs, constructed according to rule $3.3(3.4)$ for $\xi \neq 0(\xi=0)$, while only the contribution of the "vacuum graphs" appears in the denominator, drawn according to rule $3.1(3.2)$ for $\xi \neq 0$ $(\xi=0)$; all the contributions are calculated according to rule $3.5(3.6)$ for $\xi \neq 0(\xi=0)$. Following the same arguments of Ref. 2 we conclude that the denominator in Eq. (3.25) cancels all the numerator graphs with "vacuum parts" (i.e., subgraphs which have no external lines and are disconnected from the rest of the graph). The rule for the calculation of the GF is then given by the following.

Theorem 3.3. The Green's function $G(1,2, \ldots, r ; \xi)$ is the sum of all the contributions calculated according to rule 3.5 (3.6) of all the topologically distinct and vacuumfree graphs, drawn according to rule 3.3 (3.4) for $\xi \neq 0$ $(\xi=0)$.

\section{Diagrammatic rules in imaginary frequency and reciprocal space $(\xi=0)$}

We shall first consider the Fourier transform with respect to time of

$G\left(\gamma_{1}, \tau_{1} ; \ldots ; \gamma_{n}, \tau_{n}\right)=\left\langle\left[\hat{Y}\left(\gamma_{1}, \tau_{1}\right) \cdots \hat{Y}\left(\gamma_{n}, \tau_{n}\right)\right]_{+}\right\rangle_{\mathcal{H}}$

where

$$
\hat{Y}(\gamma, \tau)=\exp (\tau \mathcal{H}) Y_{\gamma} \exp (-\tau \mathcal{H})
$$

is defined for $\beta \geq \tau \geq 0$. Besides the Fermi-like operators $Y_{\gamma}$ that appear in $H_{h}$, we shall also consider Bose-like
Hubbard operators that do not change the number of electrons. We shall use the same notation for both types of $f$ operators but, as discussed before, we set $u=1$ for all Bose-like operators and do not put any restriction on the states $|a\rangle$ and $|b\rangle$ when we sum over all the corresponding transitions $\alpha=(b, a)$.

To Fourier transform the GF, it is essential that they obey the boundary condition

$$
\begin{aligned}
& \left\langle\left[\hat{Y}\left(\gamma_{1}, \tau_{1}\right) \cdots \hat{Y}\left(\gamma_{j}, \tau_{j}=\beta\right) \cdots \hat{Y}\left(\gamma_{n}, \tau_{n}\right)\right]_{+}\right\rangle_{\mathcal{H}} \\
& \quad= \pm\left\langle\left[\hat{Y}\left(\gamma_{1}, \tau_{1}\right) \cdots \hat{Y}\left(\gamma_{j}, \tau_{j}=0\right) \cdots \hat{Y}\left(\gamma_{n}, \tau_{n}\right)\right]_{+}\right\rangle_{\mathcal{H}}
\end{aligned}
$$

with respect to all the operators $\hat{Y}\left(\gamma_{1}, \tau_{1}\right) \cdots \hat{Y}\left(\gamma_{n}, \tau_{n}\right)$, where the - $(+)$ corresponds to a Fermi-like (Bose-like) operator $\hat{Y}\left(\gamma_{j}, \tau_{j}\right)$. In the proof of Eq. (3.28) it is necessary to have an even number of Fermi-like operators, a property that is not satisfied when $\xi \neq 0$, and for this reason we shall consider only $\xi=0$ in the present section. Another property that fails when $\xi \neq 0$ is the system invariance against lattice translations.

When Eq. (3.28) is satisfied for all the variables and $H$ does not depend on $\tau$, we can treat the GF as periodic (antiperiodic) with period $\beta$ in $\tau$, for all Bose-like (Fermilike) operators $Y(\gamma, \tau)$, and we then write

$$
\begin{aligned}
&\left\langle\left[\hat{Y}\left(\gamma_{1}, \tau_{1}\right) \cdots \hat{Y}\left(\gamma_{n}, \tau_{n}\right)\right]_{+}\right\rangle_{\mathcal{H}} \\
&=\beta^{-\frac{n}{2}} \sum_{\omega_{1} \cdots \omega_{n}}\left\langle\left[\hat{Y}\left(\gamma_{1}, \omega_{1}\right) \cdots \hat{Y}\left(\gamma_{n}, \omega_{n}\right)\right]_{+}\right\rangle_{\mathcal{H}} \\
& \quad \times \exp \left[-i\left(\omega_{1} \tau_{1}+\cdots+\omega_{n} \tau_{n}\right)\right] .
\end{aligned}
$$

The frequencies $\omega_{j}$ are different for the two types of operators $Y_{\gamma}$ :

$\omega_{j}=\frac{\pi \nu_{j}}{\beta}, \quad$ where $\begin{cases}\nu_{j}=0, \mp 2, \mp 4 \cdots & \text { (Bose-like) } \\ \nu_{j}=1, \mp 3, \mp 5 \cdots & \text { (Fermi-like) } .\end{cases}$

The notation of the Fourier coefficients in Eq. (3.29) is purely symbolic, because the $\tau$-ordering $(\cdots)_{+}$has no meaning there.

To Fourier transform the spatial dependence one has to remember that the $c$ operators are already in reciprocal space, so it is only necessary to transform the $f$ operators. For a GF with $r$ operators of the $f$ type (Fermi-like or Bose-like) and $n-r$ operators of the $c$ type we write in an abbreviated notation 


$$
\begin{aligned}
\left\langle[\hat{Y}(f, \tau ; 1) \cdots \hat{Y}(f, \tau ; r) \hat{Y}(c, \tau ; r+1) \cdots \hat{Y}(f, \tau ; n)]_{+}\right\rangle_{\mathcal{H}} & \\
= & \beta^{-\frac{n}{2}} N_{s}^{-\frac{r}{2}} \sum_{\vec{k}_{1} \cdots \vec{k}_{r}} \sum_{\omega_{1} \cdots \omega_{n}} \exp \left[-i\left(\vec{k}_{1} u_{1} \cdot \vec{R}_{1}+\cdots+\vec{k}_{r} u_{r} \cdot \vec{R}_{r}\right)-i\left(\omega_{1} \tau_{1}+\cdots+\omega_{n} \tau_{n}\right)\right] \\
& \quad\left\langle\left\langle[\hat{Y}(f, \omega ; 1) \cdots \hat{Y}(f, \omega ; r) \hat{Y}(c, \omega ; r+1) \cdots \hat{Y}(c, \omega ; n)]_{+}\right\rangle_{\mathcal{H}},\right.
\end{aligned}
$$

where $R_{s}$ is the position of site $j_{s}, \hat{Y}(f, \tau ; s)=\hat{Y}\left(f ; j_{s}, \alpha_{s}, u_{s}, \tau_{s}\right), \hat{Y}(c, \tau ; s)=\hat{Y}\left(c ; \vec{k}_{s}, \sigma_{s}, u_{s}, \tau_{s}\right)$, and we substitute the $\tau$ by $\omega$ in $\hat{Y}(f, \omega ; s)$ and $\hat{Y}(c, \omega ; s)$. With the same notation, the inverse relation is then

$$
\begin{aligned}
\langle[\hat{Y}(f, \omega ; 1) & \left.\cdots \hat{Y}(f, \omega ; r) \hat{Y}(c, \omega ; r+1) \cdots \hat{Y}(c, \omega ; n)]_{+}\right\rangle_{\mathcal{H}} \\
= & \beta^{-\frac{n}{2}} N_{s}^{-\frac{r}{2}} \sum_{j_{1} \cdots j_{r}} \int_{0}^{\beta} d \tau_{1} \cdots \int_{0}^{\beta} d \tau_{n} \exp \left[+i\left(\vec{k}_{1} u_{1} \cdot \vec{R}_{1}+\cdots+\vec{k}_{r} u_{r} \cdot \vec{R}_{r}\right)+i\left(\omega_{1} \tau_{1}+\cdots+\omega_{n} \tau_{n}\right)\right] \\
& \times\left\langle[\hat{Y}(f, \tau ; 1) \cdots \hat{Y}(f, \tau ; r) \hat{Y}(c, \tau ; r+1) \cdots \hat{Y}(f, \tau ; n)]_{+}\right\rangle_{\mathcal{H}} .
\end{aligned}
$$

The present definition is slightly different from Hubbard's, ${ }^{2}$ because we include the parameter $u= \pm 1$ into the spatial part of the exponential in Eqs. (3.31) and (3.32). The parameter $u$ (defined in the sentences after rule 3.4) was convenient to organize our calculation, but we did not use it in the temporal part of the exponential because it was not particularly useful there.

From the invariance under time translation (i.e., $\mathcal{H}$ does not depend on $\tau$ ) one can show that the GF in Eq. (3.32) vanishes unless

$$
\omega_{1}+\omega_{2}+\cdots+\omega_{n}=0
$$

To prove the corresponding property for the wave vectors $\vec{k}_{j}$ in Eq. (3.32), it is necessary to transform first the $c$ operators into the Wannier representation

$$
C_{j \sigma}^{\dagger}=\frac{1}{\sqrt{N_{s}}} \sum_{\vec{k}} \exp \left(-i \vec{k} \cdot \vec{R}_{j}\right) C_{\vec{k} \sigma}^{\dagger} .
$$

Substituting Eq. (3.34) into the GF in the right-hand side of Eq. (3.32) and employing the invariance under lattice translation, one finds that the GF in Eq. (3.32) vanishes unless

$$
\vec{k}_{1} u_{1}+\vec{k}_{2} u_{2}+\cdots+\vec{k}_{n} u_{n}=0 .
$$

It is clear that the relations in Eqs. (3.31) and (3.32) can be also employed for the corresponding cumulant averages. When $H_{h}=0$ many $Y_{\gamma}$ are statistically independent, and the only cumulants left in rule 3.6 must either have all their $Y_{\gamma}$ of the $f$ type and at the same site, or else have all of the $c$ type and have the same $\vec{k}$ (and same $\sigma$ when $\mathcal{H}_{0}$ is spin independent). Because of the invariance of the system under lattice translations, the local cumulants that appear in rule 3.62 (a) are independent of the site position and it is not necessary to take their spatial Fourier transform; on the other hand, the $Y_{\gamma}$ of the $C$-electron cumulants of rule $3.62\left(\mathrm{~b}^{\prime}\right)$ have already been transformed. From these two facts it follows that to obtain the Fourier transformed version of rule 3.6 it would be sufficient to apply only the transformation from time to frequency [cf. Eq. (3.29)] to the cumulants in that rule.

To set the notation we write

$$
\begin{aligned}
& \left\langle\left[Y\left(f ; j, \alpha_{p}, u_{p}, \tau_{p}\right) \cdots Y\left(f ; j, \alpha_{1}, u_{1}, \tau_{1}\right)\right]_{+}\right\rangle_{c} \\
& =\beta^{-\frac{p}{2}} \sum_{\omega_{1} \cdots \omega_{p}} \exp \left[-i\left(\omega_{1} \tau_{1}+\cdots+\omega_{p} \tau_{p}\right)\right] \\
& \quad \times\left\langle\left[Y\left(j, \alpha_{p}, u_{p}, \omega_{p}\right) \cdots Y\left(j, \alpha_{1}, u_{1}, \omega_{1}\right)\right]_{+}\right\rangle_{c}
\end{aligned}
$$

and

$$
\begin{aligned}
& \left\langle\left[Y\left(c ; \vec{k}, \sigma_{2},-u_{2}, \tau_{2}\right) Y\left(c ; \vec{k}, \sigma_{1}, u_{1}, \tau_{1}\right)\right]_{+}\right\rangle_{c} \\
& =\beta^{-1} \sum_{\omega_{1} \omega_{2}} \exp \left[-i\left(\omega_{1} \tau_{1}+\omega_{2} \tau_{2}\right)\right] \\
& \quad \times\left\langle\left[C\left(\vec{k}, \sigma_{2},-u_{2}, \omega_{2}\right) C\left(\vec{k}, \sigma_{1}, u_{1}, \omega_{1}\right)\right]_{+}\right\rangle_{c} .
\end{aligned}
$$

Note that the invariance under time translation guarantees that Eq. (3.33) would be satisfied for the frequency dependent cumulants of Eqs. (3.36) and (3.37). To proceed with the transformation of rule 3.6, we use the prescription given in the previous sections to express the GF in the rhs of Eq. (3.32) as a sum of terms, each corresponding to some graph. In the contribution of each graph one introduces Eqs. (3.36) and (3.37) and then performs explicitly all the integrations over $\tau$ and all the unrestricted summations over the sites $j$.

In each integration over $\tau$ there are two possibilities: the $\tau$ corresponds either to an external operator or else to an internal line. When the $\tau_{j}$ corresponds to an external operator $Y\left(\gamma_{j}, \tau_{j}\right)$, Eq. (3.32) provides the integration, and the integrand has two factors: one $\exp \left(i \omega_{j} \tau_{j}\right)$ 
from Eq. (3.32) and another $\exp \left(-i \omega_{s} \tau_{j}\right)$ from applying Eqs. (3.36) and (3.37) to the cumulant of rule 3.6 that contains the external operator $Y\left(\gamma_{j}, \tau_{j}\right)$. As both $\omega_{j}$ and $\omega_{s}$ are of the same type [cf. Eq. (3.30)], the integral vanishes unless $\omega_{j}=\omega_{s}$, and from the sum over all the $\omega_{s}$ in Eqs. (3.36) and (3.37) only the external frequency $\omega_{j}$ remains.

When the $\tau_{s}$ belongs to an internal line, the integration comes from the perturbation expansion [cf. Eq. (3.11)], and the integrand is $\exp \left[-i\left(\omega_{s}+\omega_{s}^{\prime}\right) \tau_{s}\right]$ where $\omega_{s}$ and $\omega_{s}^{\prime}$ come from expanding with Eq. (3.36) or Eq. (3.37) the two cumulants of rule 3.6 that contain the $C$ operator and the $X$ operator of the internal line. The integration is again zero unless $\omega_{s}+\omega_{s}^{\prime}=0$, and one can then associate only one of these two frequencies to the internal line in the transformed rules.

With the spatial part we have only applied the Fourier transformation to the external $X$ operators in Eq. (3.32), and it is then convenient to write explicitly the dependence with $R_{j}$ of the coupling constants of Eq. (3.20):

$$
v(j, \alpha, \vec{k}, \sigma, u)=V(\alpha, \vec{k}, \sigma, u) N_{s}^{-\frac{1}{2}} \exp \left(i u \vec{k} \cdot \vec{R}_{j}\right)
$$

It is then clear that the perturbation expansion provides a factor $\exp \left(i u \vec{k} \cdot \vec{R}_{j}\right)$ to each internal line, with the same $u$ and $R_{j}$ that are associated to the corresponding $X$ operator [cf. Eq. (3.11) and rule 3.6], as well as an unrestricted sum over all the sites $j$. All the $\vec{k}$ satisfy periodic boundary conditions and the summation over sites would vanish unless the sum of all the products $u \vec{k}$ at the site is zero [i.e., Eq. (3.35) is satisfied at each vertex]. After all these considerations we give the transformed rules without further discussion.

Rule 3.7. To calculate the contribution of a diagram obtained from rule 3.4.

(1) Assign to each internal line a momentum $\vec{k}_{s}$, a frequency $\omega_{s}$, and an index $\pm u_{s}$. Assign dummy labels $\alpha_{s}$ and $\pm u_{s}$ to the $X$ operators at the FV side of the internal line, and dummy labels $\vec{k}_{s}, \sigma_{s}$, and $\mp u_{s}$ to the $C$ operators at the CV side. Use $+u_{s}$ and $+\omega_{s}$ at the side of the edge to which the arrow points (cf. item iv of rule 3.4) and $-u_{s}$ and $-\omega_{s}$ to the opposite side.

Assign to the external lines the labels of the corresponding external operators, namely the momentum $\vec{k}_{s}$, frequency $\omega_{s}$, index $u_{s}$, and also the transition $\alpha_{s}=$ $\left(b_{s}, a_{s}\right)$ for $X$ operators and the spin component $\sigma_{s}$ for the $C$ operators (we use always $+u_{r}$ and $+\omega_{r}$ for the external lines).

(2) Form the product of the following factors.

(a) For each FV with lines $s=1,2, \ldots, p$ running to that vertex (both internal and external) the factor

$$
\begin{aligned}
N_{s} \delta\left( \pm u_{p} \vec{k}_{p} \pm \cdots \pm u_{2} \vec{k}_{2} \pm u_{1} \vec{k}_{1}\right) \\
\quad \times\left\langle\left[X\left(j, \alpha_{p}, \pm u_{p}, \pm \omega_{p}\right) \cdots X\left(j, \alpha_{1}, \pm u_{1}, \omega_{1}\right)\right]_{+}\right\rangle_{c}
\end{aligned}
$$

where $\vec{k}_{s}, \omega_{s}, \alpha_{s}$, and $u_{s}$ are the momentum, frequency transition, $\alpha_{s}=\left(b_{s}, a_{s}\right)$, and index $u_{s}$ labels of the $X$ operators associated to line $s$ (always $+u_{s}$ and $+\omega_{s}$ for the external lines).

(b) For each CV a factor

$$
\left\langle\left[C\left(\vec{k}_{2}, \sigma_{2},-u_{2},-\omega_{2}\right) C\left(\vec{k}_{1}, \sigma_{1}, u_{1}, \omega_{1}\right)\right]_{+}\right\rangle_{c},
$$

where $\vec{k}_{1}, \sigma_{1}, u_{1}$, and $\omega_{1}$ are the parameters of the edge with the arrow pointing towards the CV. As we discussed before, this cumulant vanishes unless $\vec{k}_{1}=\vec{k}_{2}, u_{1}=u_{2}$, and $\omega_{1}=\omega_{2}$. When the Bloch states $|\vec{k}, \sigma\rangle$ are eigenstates of $\mathcal{H}_{0}$, we have also $\sigma_{1}=\sigma_{2}$ and the factor above is equal to

$$
\frac{1}{i \omega_{1}+u_{1} \epsilon\left(\vec{k}_{1}, \sigma_{1}\right)} \delta\left(\vec{k}_{1}, \vec{k}_{2}\right) \delta\left(u_{1}, u_{2}\right) \delta\left(\sigma_{1}, \sigma_{2}\right) \delta\left(\omega_{1}, \omega_{2}\right),
$$

where the parameters with subindex 1 correspond again to the edge with the arrow pointing towards the $\mathrm{CV}$ (when the outgoing line is external with given $u$ and $\omega$, we set $-u_{2}=u$ and $\left.-\omega_{2}=\omega\right)$.

(c) A factor $V(\alpha, \vec{k}, \sigma, \pm u)$ for each internal line with labels $\alpha, \pm u$ at the FV site and labels $\vec{k}, \sigma$, and $\mp u$ at the CV side.

(d) A factor \pm 1 determined by the rules in Appendix C.

(e) A factor $1 / g$ determined by the rules in Appendix

(f) A factor $1 / \sqrt{N_{s}}$ for each external line joining a FV. (3) Sum the resulting product with respect to the following.

(a) The momenta $\vec{k}_{s}$, the frequencies $\omega_{s}$, and the indices $u_{s}$ of all the internal edges. Divide each sum over momenta into $\sqrt{N_{s}}$.

(b) The labels $\alpha_{s}$ of the $X$ operators at the FV side of all internal lines.

(c) The label $\sigma_{s}$ of the $C$ operators at the CV side of all internal lines.

Two points should be stressed. (i) The frequencies of each local cumulant in rule 3.72 (a) satisfy Eq. (3.33), thus reducing by one the number of frequency summations at each FV. (ii) The rules are also valid for vacuum graphs, and are employed to calculate the GCP with the linked cluster theorem

\section{APPLICATION: CALCULATION OF THE OCCUPATION NUMBERS}

In this section we discuss the results obtained for the PAM in the limit of infinite electronic repulsion $(U=\infty)$, in which the state with two electrons at the same site is always empty. We shall consider two families of diagrams: the simplest one has all possible diagrams with only second order cumulants [cf. Figs. 3(a)-3(c)] and it shall be called the chain approximation (CHA). In the second approximation we also consider diagrams with fourth order cumulants at an infinite number of sites [cf. Figs. 4(a) $-4(c)$ ], and at each site with a fourth order cu- 
a)

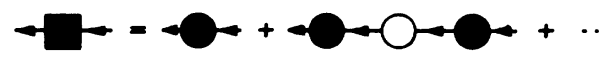

b)

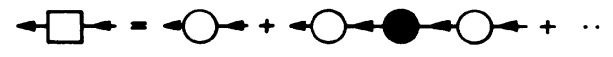

c)

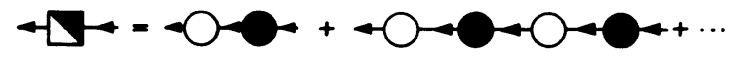

FIG. 3. The diagrams for the CHA. (a) The family of diagrams that give the $f$ electron GF. (b) Same as (a) for the $c$ electrons. (c) Family of diagrams that give a "mixed" GF. We employ the symbol at the left side of (a), (b), and (c) to indicate the infinite diagrams at their right.

mulant a loop of any length is attached: we shall call this the multiple loop approximation (MLA).

We shall consider two cases. (i) The atomic limit, in which the conduction electrons have zero bandwidth because they are localized. (ii) An extended conduction band. We shall only consider a rectangular band: this simplifies the analytic calculations but still shows the features of the model. We shall use a purely local hybridization interaction: $V_{\vec{k}}=V$.

Within these approximations we have calculated the one-particle GF, and obtained with them the occupation number per site and per spin of the $f$ electrons $n_{f, j, \sigma}=$ $\left\langle X_{j, \sigma \sigma}\right\rangle$ and of the $c$ electrons $n_{c, j, \sigma}=\left\langle C_{j, \sigma}^{\dagger} C_{j, \sigma}\right\rangle$, where we have employed the Wannier representation of the $C$ operators [cf. Eq. (3.34)]. One difficulty that appeared immediately in the CHA was the lack of completeness in the space of the local states when $\mu$ is in the neighborhood of $E_{f}$. As we assume a paramagnetic and uniform system in the present paper, this completeness is

a)

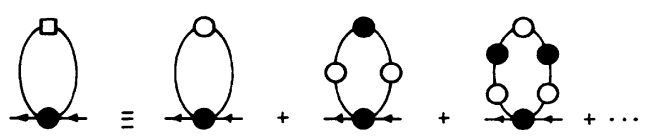

b)

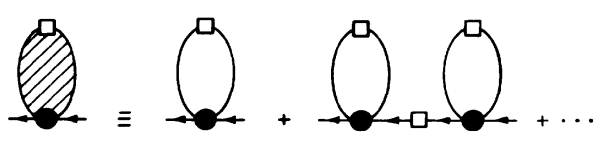

c)

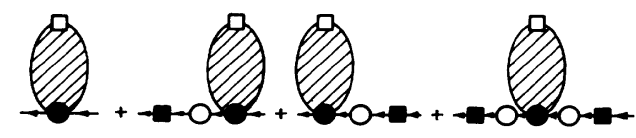

FIG. 4. The multiple loop approximation (MLA). (a) The family of diagrams with only one fourth order cumulant. (b) Diagrams with infinite fourth order cumulants. (c) The diagrams that give the MLA correction to the GF in the CHA. They should be added to the diagrams in Fig. 3(a). expressed by the relation $2 n_{f}+n_{0}=1$. A procedure that has been used ${ }^{13}$ to satisfy the local completeness was to calculate self-consistently the $n_{f}$ and $n_{0}$ obtained from the approximate GF, employing $2 n_{f}+n_{0}=1$ as a further condition, but although this method is valid in the CHA, it does not work in general. ${ }^{15}$ Although the self-consistent calculation of averages is perfectly valid for the method of equations of motion, the unperturbed ones ( $n_{0}^{0}$ and $n_{f}^{0}$ in the present case) should be used as parameters to obtain the approximate GF in the spirit of the present perturbation calculation.

We have recently made an important advance in the solution of the completeness problem: we have found that by adding certain diagrams to those of the CHA and of other families of diagrams, the completeness in the $f$ space is automatically attained, both for the wide band and in the atomic limit. We are working in this problem, and the results shall be presented in a forthcoming paper.

Another difficulty appears first in the MLA: as usual we make the analytic continuation of the Fourier coefficients of the Matsubara GF to the upper and lower half-planes of the complex frequency $z$, and the resulting function $\bar{G}_{\sigma \sigma^{\prime}}^{f f}(\vec{k}, z)$ is minus the Fourier transform of the real time GF. This function is always analytic off the real axis for the CHA, but for a wide range of parameters the MLA gives a nonanalytic GF in that region. Decoupling the equations of motion for the GF has been shown to give similar singularities for the Hubbard model. ${ }^{16}$ In both cases, the usual calculations with the corresponding real time GF [which we shall call $\operatorname{MLA}\left(C_{2}\right)$ (Refs. 15 and 17)] give unphysical results, but we have found that sensible values of static properties can be obtained by direct calculation from the Matsubara GF, or what is the same, by employing $\bar{G}_{\sigma \sigma^{\prime}}^{f f}(\vec{k}, z)$ in the usual way but integrating along a circuit $C_{3}$ in the complex $z$ plane that includes its singularities off the real axis [we shall use $\operatorname{MLA}\left(C_{3}\right)$ to refer to this method]. We have found that with both methods completeness is not satisfied for $\mu \simeq E_{f}$, but that $\operatorname{MLA}\left(C_{2}\right)$ gives $n_{0}+2 n_{f} \neq 1$ even for $\mu \rightarrow \infty$ while $\operatorname{MLA}\left(C_{3}\right)$ satisfies completeness in this region. For some parameter regimes, the singularities off the real axis are present in the MLA even when $T \longrightarrow \infty$ and the $\operatorname{MLA}\left(C_{3}\right)$ always gives the best results, showing that $\operatorname{MLA}\left(C_{2}\right)$ clearly lacks the contribution of the singularities off the real axis to give the correct dependence when $\mu \rightarrow \infty$.

Typical results of $n_{c}$ as a function of the chemical potential $\mu$ in the atomic limit are shown in Fig. 5 for $E_{f}=-0.05, V=0.1$, and $T=0.025$. We plot the exact curve as well as the approximate ones calculated with the CHA and also with the $\operatorname{MLA}\left(C_{3}\right)$ and the $\operatorname{MLA}\left(C_{2}\right)$. As completeness is satisfied in the space of the $c$ electrons, these results single out the effect of the singularities that are off the real axis. The most interesting curve is the $\operatorname{MLA}\left(C_{2}\right)$, because it shows that even for $\mu \rightarrow \infty, n_{c}$ reaches an asymptotic value of $n_{c}=0.5$, i.e., one-half of the correct asymptotic value $n_{c}=1$ shown by both the CHA and the $\operatorname{MLA}\left(C_{3}\right)$ when $\mu \rightarrow \infty$. The reason for this failure is that the GF singularities that are off the real axis account for one-half of the maximum number of $c$ electrons, emphasizing the need of including those 


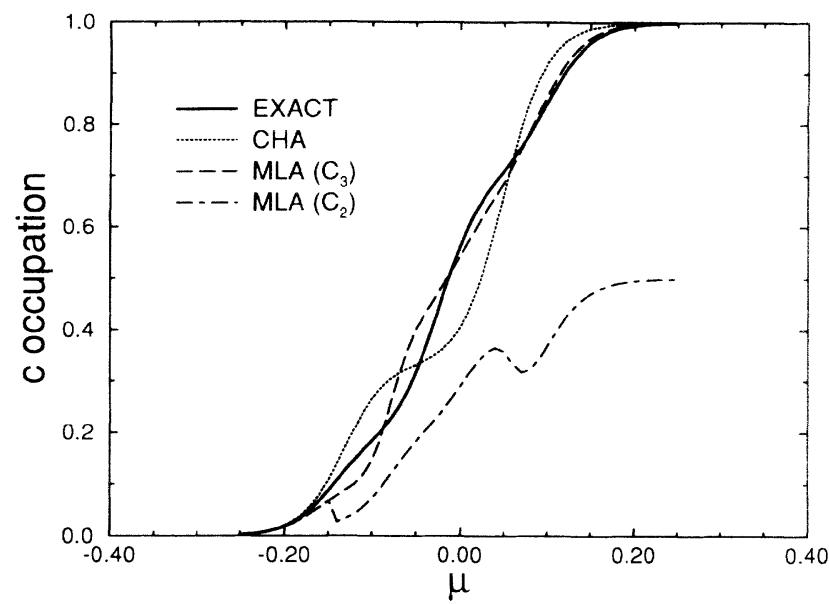

FIG. 5. Plot of the occupation numbers $n_{c}$ for the $c$ electrons as a function of the chemical potential $\mu$, in the atomic limit. They are calculated for $E_{f}=-0.05, E_{0}=0, V=0.1$, and $T=0.025$, measured in the same energy units. The different approximations are indicated in the graph.

singularities in the calculation.

We have made extensive calculations ${ }^{15}$ in the atomic limit with the $\operatorname{MLA}\left(C_{3}\right)$ and have found that the values of statistical properties like the occupation number compare moderately well with the exact result.

We have also made calculations ${ }^{15}$ for the PAM with a rectangular band centered at the origin. As in the atomic limit, we find singularities off the real axis in the $\mathrm{GF}$, because $\mathrm{MLA}\left(C_{3}\right)$ and $\operatorname{MLA}\left(C_{2}\right)$ give rather different results. These singularities are branch cuts that are symmetrically located with respect to the real axis. In spite of this undesirable property of the GF, static properties like the occupation numbers give fairly good results for the $\operatorname{MLA}\left(C_{3}\right)$, but it is clear that in the presence of those singularities we cannot hope to obtain reliable spectral densities and dynamical properties, because they require the analytic continuation of the Matsubara GF to the real axis. Another aspect to emphasize is that we have also found that the $\operatorname{MLA}\left(C_{3}\right)$ gives nearly perfect completeness except for a small region in the neighborhood of $\mu \sim E_{f}$, and the discrepancy is clearly much less pronounced than in the atomic case.

In Fig. 6 we plot $n_{f}$ versus $\mu$ for a band of width $2 W=\pi$, for the same parameters of Fig. 5 but for $T=0.01$ rather than $T=0.025$. The differences between the CHA and the $\operatorname{MLA}\left(C_{3}\right)$ for statical properties like the occupation number are fairly small for the parameters employed, seeming to indicate that the convergence in the band case is much better than in the atomic case.

We shall make a brief study of the spectral densities that are obtained, bearing in mind that they give unphysical results when there are singularities off the real axis. The unperturbed spectral density of the $f$ electron is a $\delta$ function located at $\epsilon_{f}=E_{f}-\mu$, but it becomes a band in the CHA, opening a gap around $\epsilon_{f}$ as shown in Fig. 7(a). In the MLA the boundaries of the gap are not abrupt but rounded. Two peaks that are very close together appear in the gap, and their position changes with $\mu$. At rather low $T(T<0.001)$ and for $\epsilon_{f} \sim 0$ the

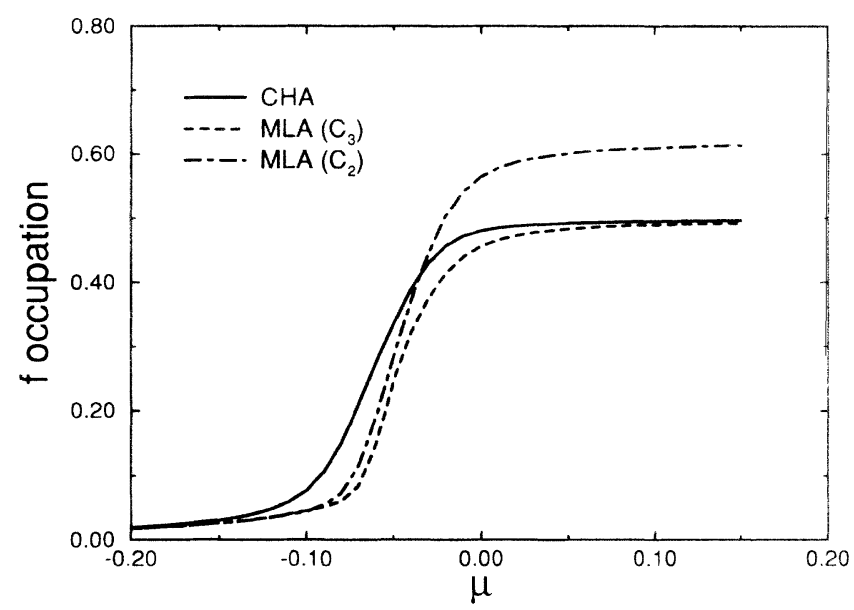

FIG. 6. Plot of the number $n_{f}$ of $f$ electrons as a function of the chemical potential $\mu$, for the same parameters of Fig. 5 but for a band of width $2 W=\pi$. The approximations employed are indicated in the figure.
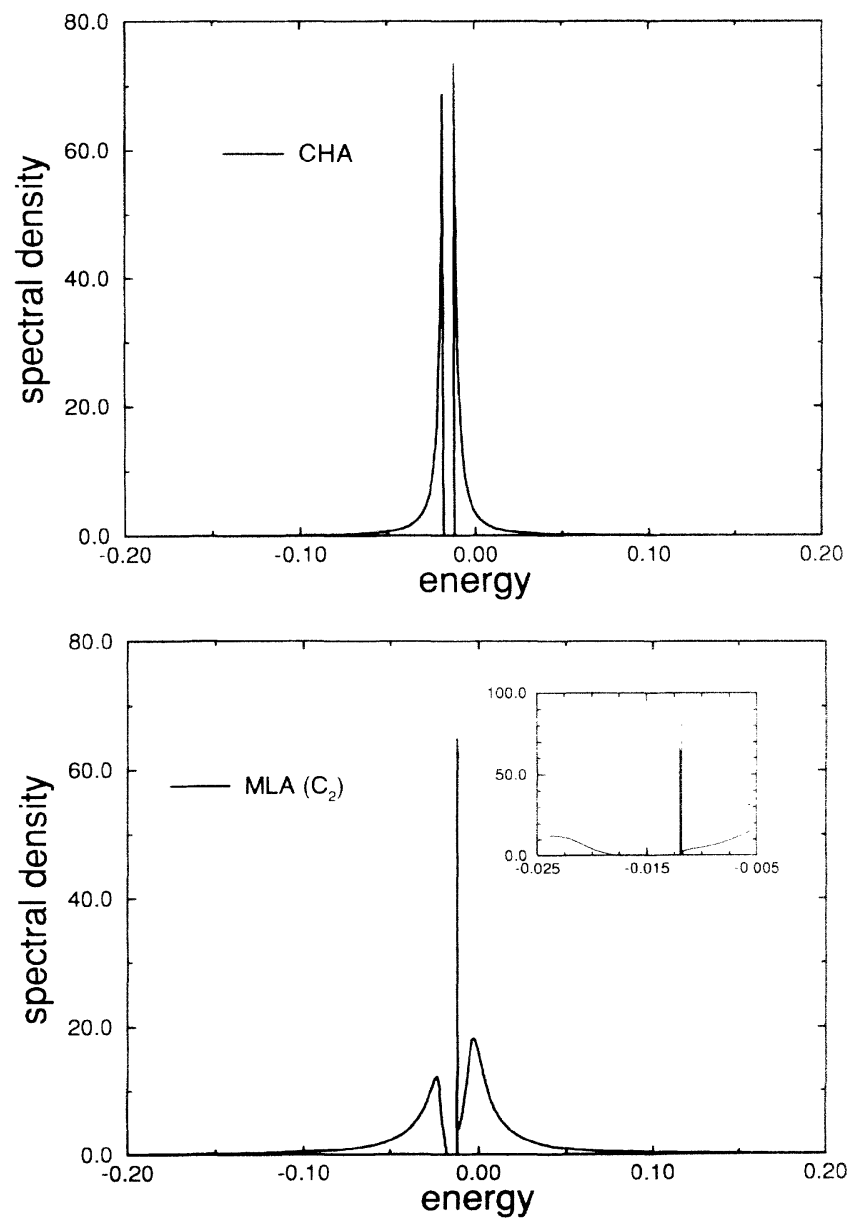

FIG. 7. Plot of the spectral density of the $f$ electrons as a function of $\omega$ for a band of width $2 W=\pi$ and for $E_{f}=-0.05$, $E_{0}=0, V=0.1, T=0.001$, and $\mu=-0.035$ measured in the same energy units. For these parameters, the GF are analytic off the real axis. (a) The CHA: a gap appears at $\omega \sim \epsilon_{f}=E_{f}-\mu$. (b) The MLA; the inset shows the detail of the double peak inside the gap. 
GF are always analytic off the real axis, and the plot in Fig. 7(b) corresponds to this situation; in the inset we show that the peak inside the gap is double.

As it is possible to trace the terms that correspond to spin-flip in the fourth order cumulant, we have found that the two peaks inside the gap originate exclusively from this type of interaction. These two peaks are not the Kondo resonance, because they are always inside the gap rather than close to $\mu$, and because they do not disappear even at very high $T$.

Although the MLA includes infinite fourth order cumulants that contain the spin-flip transitions that are the basis of the Kondo effect, ${ }^{12}$ the Kondo resonance was not observed in this approximation. This seems to indicate that the MLA misses some important ingredients to give an adequate description of the PAM in the Kondo region, and we are further studying this problem by considering possible extensions of the families of diagrams that appear in the MLA, as well as $\Phi$-derivable approximations with only fourth order cumulants.

\section{CONCLUSIONS}

In the present work we have extended the cumulant expansion derived by Hubbard ${ }^{2}$ for his model, to a system in which localized electrons with strong correlation are hybridized with a band of uncorrelated conduction electrons. This treatment describes the PAM, but it can be also employed to study a system in which the localized electrons have several strongly correlated configurations with a rather arbitrary distribution of levels. This extension is of interest to study systems with anomalous rare earths like Eu and Tm.

The diagrammatic expansion we derived is also valid for nonzero external (Grassmann) fields $\xi$, and we have obtained a "linked cluster" expansion (theorem 3.2) for the GCP. This extension shall be employed in a future publication on the " $\Phi$-derivable" approximation., ${ }^{3,7}$ The rules for the construction in real space and imaginary time of the $n$ th-order "vacuum" diagrams (rules 3.1 and 3.2) and of the $n$ th-order "rooted" diagrams (rules 3.3 and 3.4) are given for both the $\xi \neq 0$ and $\xi=0$ cases, as well as the calculation of their corresponding contributions (rules 3.5 and 3.6). We also give for the GF the method that corresponds to the linked cluster expansion of the GCP (theorem 3.3), and we consider GF with any numiber of Fermi-type operators and of a special type of Bose operators (those that do not change the number of electrons). The Fourier transformation of these rules to imaginary frequency and reciprocal space is only presented for the $\xi=0$ case (rule 3.7). These rules are the basis for the calculation of static and dynamic properties for both the PAM and for the many other interesting problems described by the extended model.

It has been already emphasized ${ }^{7}$ that one strength of the cumulant method is that the lattice sums are unrestricted, so that there are no difficulties with excluded sites. We have employed this property to obtain a version of the diagrammatic expansion of the GCP and of the GF that is Fourier transformed from real to reciprocal space.
It is interesting to compare the cumulant expansion for the PAM with another expansion around the atomic limit that was developed in a series of papers by Keiter, Kimball, and Grewe (KKG) ${ }^{18-20}$ and that also employs Hubbard operators. The two methods start with the standard perturbation expansion of the partition function, leading to the same $n$ th-order term written in our Eq. (3.8) [cf. Eq. (3) in Ref. 19]. From this point onwards, the two expansions differ, and the KKG method splits the averages of the $2 n$ operators $Y(l)$ [cf. Eq. (3.11)] as the product of several averages: one containing all the $c$ operators, and the remaining ones being one for each site. Each of these last averages at a given site is taken over all the $f$ operators that are in the starting average at that site. Employing Wick's theorem for the $c$-operators average, KKG were able to emphasize the interpretation of all the terms in the expansion as elementary excitation processes, but at the cost of having an excluded volume problem that is very hard to treat rigorously, ${ }^{21}$ and sacrificing the linked cluster expansion. ${ }^{19}$ These difficulties do not appear in the cumulant method, because employing theorem 3.1 the averages in Eq. (3.11) are decomposed into a sum of products of cumulant correlations.

The KKG method employs infinite resummations that lead to a scheme of the Brillouin-Wigner type. ${ }^{18,19,22}$ In the impurity case a successful development of the KKG method was the noncrossing approximation (NCA) that considers a particular infinite family of diagrams, ${ }^{23-25}$ and leads to a system of two coupled integral equations. This technique has been extended to the PAM, ${ }^{26,27,21}$ and applied to the uncorrelated problem $(U=0)$ by Grewe, ${ }^{26}$ who called it the "resonant level model." In the cumulant expansion, the CHA already gives the exact solution, but in the NCA Grewe ${ }^{26}$ has shown that the spectral density shows a spurious residual weight near the Fermi level that is particularly noticeable at low $T$ in the intermediate valence (IV) region. ${ }^{26}$

The calculation of the PAM with the KKG method employs the solution of the impurity problem. This has the advantage of carrying over to the lattice properties like the Kondo resonance of the spectral density, which is already present in the impurity GF when the NCA is used. ${ }^{21}$ On the other hand, the structure of the impurity GF is completely changed in the lattice, ${ }^{26}$ and the excluded volume problem is very hard to treat rigorously. ${ }^{21}$ This situation is reversed in the cumulant method: there is no excluded site restriction, and the impurity GF is not employed in the lattice treatment. From these considerations, we believe that the cumulant expansion is more natural than the KKG method for studying the lattice problems, with the disadvantage that it is more difficult to obtain the Kondo resonance, as discussed in Sec. IV.

To conclude, let us point out that the present work follows closely the derivation presented by Hubbard in his original paper, ${ }^{2}$ but with the following differences.

(1) In Hubbard's work, the unperturbed Hamiltonian $H_{0}$ contains only localized electrons. One could use the same formalism to study the PAM by splitting the states at each site in two groups: one for the $f$ electrons and another for the $c$ electrons. The hybridization would then be described by a "hopping" between the two groups, 
and the conduction band would be obtained through the hopping of the $c$ electrons between different sites. As the interesting case for the PAM is that of a wide conduction band, it is better to include the $c$-electron hopping into $H_{0}$. We have been able to extend Hubbard's derivation to this model, because its essential property is not the locality of the electrons but rather their statistical independence with respect to the statistical probability in the unperturbed system (cf. Sec. I).

(2) Because of the inclusion of a conduction band in $H_{0}$, we had to introduce the $c$-electron vertices into the diagrams and reformulate all the rules for the diagrammatic calculation.

(3) Hubbard uses the Grassmann fields $\xi$ in his derivation, but gives the diagrammatic rules only for $\xi=0$. As discussed above, we shall need diagrams with $\xi \neq 0$ to study the " $\Phi$-derivable" approximation in a future work. We have shown (cf. Sec. III A) that by an adequate choice of " $\xi$ averages" [cf. Eq. (3.12)] it is possible to derive a diagrammatic expansion for $\xi \neq 0$ that is essentially the same derived for $\xi=0$, except for the rule derived from the conservation of particles, which fails when $\xi \neq 0$.

\section{ACKNOWLEDGMENTS}

The authors would like to acknowledge financial support from the following agencies: M.S.F. from CAPES-PICD, M.E.F. from FAPESP, CNPq, and FAEP(Unicamp), and G.G.M. from FAPESP and FAEP (Unicamp).

\section{APPENDIX A: THE EVOLUTION OPERATOR FOR COMPLEX TIMES}

The evolution operator for the time dependent Schrödinger equation is given by

$$
U_{r}\left(t, t^{\prime}\right)=\exp +\left\{-i \int_{t^{\prime}}^{t} d t_{1} H_{r}\left(t_{1}\right)\right\},
$$

where $H_{r}(t)$ is a time dependent Hamiltonian for the whole system, and we employ the subindex $r$ to emphasize that $t$ is the real time. The symbol exp + means $^{11}$ that we time-order all the terms in the formal expansion of the exponential, and there is a change of sign in any term of the expansion when two operators of the Fermi type must be exchanged to put them in the correct ordering.

To study finite temperature problems Matsubara ${ }^{14}$ considered complex times $\tau=i t$, and the natural extension of Eq. (A1) is

$$
U\left(\tau, \tau^{\prime}\right)=\exp +\left\{-\int_{\tau^{\prime}}^{\tau} d \tau_{1} H\left(\tau_{1}\right)\right\},
$$

where $H(\tau)=H_{r}(-i \tau)$ and in this case the time ordering means that the real variable $\tau$ increases to the left. ${ }^{11}$ We shall often use $U(\tau)=U(\tau, 0)$, and when $H(\tau)$ is independent of $\tau$, one obtains $U(\tau)=\exp (-\tau H)$, which is the statistical operator for temperature $T=1 / \tau$. To pursue the line developed by Hubbard we give some prop- erties and explicit definitions that are employed in our treatment.

Given a constant Hermitian operator $R$, we define a transformation that maps any operator $A$ into

$$
A_{R}(\tau)=\exp (\tau R) A \exp (-\tau R)
$$

and we shall use

$$
\bar{A}_{R}(\tau)=\left(A_{R}(-\tau)\right)^{\dagger}
$$

to denote the transform of $A^{\dagger}$, the Hermitian conjugate of $A$. The system Hamiltonian $H$ (in the absence of external fields) is usually $H=H_{0}+V$, where $H_{0}$ is the unperturbed part and $V$ a perturbation, and we shall use $A(\tau)=A_{H_{0}}(\tau)$ and $\hat{A}(\tau)=A_{H}(\tau)$. The operator $\hat{A}(\tau)$ then corresponds to the Heisenberg picture and $A(\tau)$ to the interaction picture of the physical quantity corresponding to $A$.

To develop the cumulant expansion we shall add an extra term to the Hamiltonian $H$ :

$$
H_{e}(\xi)=-\sum_{\gamma} \xi_{\gamma}(\tau) Y_{\tau}=H_{e}[\xi(\tau)]
$$

where the $\xi(\tau)$ are the external fields already discussed in Sec. III. The second equality emphasizes that even in the Schrödinger picture the $H_{e}(\xi)$ is a function of $\tau$ through the $\xi$ dependence. In the notation introduced above we shall use $H_{e}(\tau, \xi)$ and $\hat{H}_{e}(\tau, \xi)$ for the corresponding operators in the interaction and Heisenberg pictures, respectively, and both have a double dependence with $\tau$ : one through the picture employed, and the other through the rather arbitrary dependence of the $\xi(\tau)$. In the Schrödinger picture, we denote the total Hamiltonian in the presence of fields with $H[\xi(\tau)]=H+H_{e}[\xi(\tau)]$ (the absence of the subindex " $e$ " identifies the full Hamiltonian) and the result of applying Eq. ( A3) to $H[\xi(\tau)]$ is $H_{R}[\tau, \xi(\tau)]$.

In the presence of external fields, the evolution operator becomes

$$
U\left(\tau, \tau^{\prime}, \xi\right)=\exp +\left\{-\int_{\tau^{\prime}}^{\tau} d \tau_{1} H\left[\xi\left(\tau_{1}\right)\right]\right\}
$$

which satisfies the usual properties like

$$
U^{-1}\left(\tau, \tau^{\prime}, \xi\right)=U\left(\tau^{\prime}, \tau, \xi\right)
$$

and

$$
U\left(\tau_{1}, \tau_{2}, \xi\right) U\left(\tau_{2}, \tau_{3}, \xi\right)=U\left(\tau_{1}, \tau_{3}, \xi\right) .
$$

In the same spirit of Eq. (A3) we introduce

$$
\mathcal{S}_{R}\left(\tau, \tau^{\prime}, \xi\right)=U_{R}^{-1}(\tau) U\left(\tau, \tau^{\prime}, \xi\right) U_{R}\left(\tau^{\prime}\right)
$$

where

$$
U_{R}(\tau)=\exp (-R \tau)
$$

The $\mathcal{S}_{R}\left(\tau, \tau^{\prime}, \xi\right)$ satisfies the differential equation

$\frac{d}{d \tau} \mathcal{S}_{R}\left(\tau, \tau^{\prime}, \xi\right)=-\left\{H_{R}[\tau, \xi(\tau)]-R\right\} \mathcal{S}_{R}\left(\tau, \tau^{\prime}, \xi\right)$ 
and from Eqs. (A7) and (A8) follows

$$
\mathcal{S}_{R}^{-1}\left(\tau, \tau^{\prime}, \xi\right)=\mathcal{S}_{R}\left(\tau^{\prime}, \tau, \xi\right)
$$

and

$$
\mathcal{S}_{R}\left(\tau_{1}, \tau_{2}, \xi\right) \mathcal{S}_{R}\left(\tau_{2}, \tau_{3}, \xi\right)=\mathcal{S}_{R}\left(\tau_{1}, \tau_{3}, \xi\right)
$$

By formal integration of Eq. (A11) we obtain

$$
\mathcal{S}_{R}\left(\tau, \tau^{\prime}, \xi\right)=\exp +\left\{-\int_{\tau^{\prime}}^{\tau} d \tau_{1}\left\{H_{R}\left[\tau_{1}, \xi\left(\tau_{1}\right)\right]-R\right\}\right\}
$$

and for $R=H$ and $R=H_{0}$ we obtain

$$
\mathcal{S}_{H}(\beta, \xi) \equiv \mathcal{S}_{H}(\beta, 0, \xi)=\exp +\left\{-\int_{0}^{\beta} d \tau \hat{H}(\tau, \xi)\right\}
$$

and

$$
\begin{aligned}
\mathcal{S}_{H_{0}}(\beta, \xi) & \equiv \mathcal{S}_{H_{0}}(\beta, 0, \xi) \\
& \equiv \exp _{+}\left\{-\int_{0}^{\beta} d \tau\left[V(\tau)+H_{e}(\tau, \xi)\right]\right\},
\end{aligned}
$$

respectively, where $V(\tau)$ is $V$ in the interaction picture. Employing the properties of the "chronological" ordering one can prove that

$$
\mathcal{S}_{H_{0}}(\beta, \xi)=\left(\mathcal{S}_{H_{0}}^{0}(\beta) \mathcal{E}(\beta, \xi)\right)_{+},
$$

where

$$
\mathcal{S}_{H_{0}}^{0}(\beta)=\mathcal{S}_{H_{0}}(\beta, \xi=0)
$$

and

$$
\mathcal{E}(\beta, \xi)=\exp _{+}\left\{-\int_{0}^{\beta} d \tau H_{e}(\tau, \xi)\right\} .
$$

Equation (A17) is useful to define cumulants for nonzero $\xi$ in a way that simplifies the extension of the diagrammatic expansion of Hubbard to this situation.

From the properties above one can show that

$$
\begin{aligned}
\exp (-\beta H)\left[\hat{Y}\left(l_{1}\right) \cdots \hat{Y}\left(l_{n}\right) \mathcal{S}_{H}(\beta, \xi)\right]_{+} \\
=\exp \left(-\beta H_{0}\right)\left[Y\left(l_{1}\right) \cdots Y\left(l_{n}\right) \mathcal{S}_{H_{0}}(\beta, \xi)\right]_{+},
\end{aligned}
$$

which is valid when $0<\tau_{j}<\beta$ for all $j=1, \ldots, n$. The index $l_{j}=\left(\gamma_{j} ; \tau_{j}\right)$ includes all the indices necessary to describe the operator $Y$ as well as the complex time $\tau$, and following the notation introduced above we write

$$
Y(l)=\exp \left(\tau H_{0}\right) Y_{\gamma} \exp \left(-\tau H_{0}\right)
$$

and

$$
\hat{Y}(l)=\exp (\tau H) Y_{\gamma} \exp (-\tau H) .
$$

We introduce the average

$$
\langle A(\tau)\rangle_{R}=\frac{\operatorname{Tr}\{\exp (-\beta R) A\}}{\operatorname{Tr}\{\exp (-\beta R)\}}
$$

and we shall reserve $\langle A\rangle$ for $R=H_{0}$, i.e., for the static average of $A$ when $V=\xi=0$ (unperturbed system without external fields).

To define the GF in the presence of external fields we write

$$
\left\langle\left[\hat{Y}\left(l_{1}\right) \cdots \hat{Y}\left(l_{n}\right)\right]_{+}\right\rangle^{v \xi}
$$

$$
=\frac{\operatorname{Tr}\left\{\left[\hat{Y}\left(l_{1}\right) \cdots \hat{Y}\left(l_{n}\right) U(\beta, \xi)\right]_{+}\right\}}{\mathcal{Z}(\beta, \xi)},
$$

where $U(\beta, \xi)=U(\beta, 0, \xi)$ and

$$
\mathcal{Z}(\beta, \xi)=\operatorname{Tr}\{U(\beta, \xi)\}=\operatorname{Tr}\left\{U_{R}(\beta) \mathcal{S}_{R}(\beta, \xi)\right\}
$$

is the partition function in the presence of the external fields $\xi$. We can express the GF for $\xi \neq 0$ employing functional derivatives of the $\mathcal{Z}(\beta, \xi)$ :

$$
\left\langle\left[\hat{Y}\left(l_{1}\right) \cdots \hat{Y}\left(l_{n}\right)\right]_{+}\right\rangle^{V \xi}=\frac{1}{\mathcal{Z}(\beta, \xi)} \frac{\delta^{n} \mathcal{Z}(\beta, \xi)}{\delta \xi\left(l_{1}\right) \cdots \delta \xi\left(l_{n}\right)}
$$

and from Eqs. (A24), (A25), and (A9) we can write

$$
\left\langle\left[\hat{Y}\left(l_{1}\right) \cdots \hat{Y}\left(l_{n}\right)\right]_{+}\right\rangle^{V \xi}
$$

$$
\begin{aligned}
& =\frac{\left\langle\left[\hat{Y}\left(l_{1}\right) \cdots \hat{Y}\left(l_{n}\right) \mathcal{S}_{H}(\beta, \xi)\right]_{+}\right\rangle_{H}}{\left\langle\mathcal{S}_{H}(\beta, \xi)\right\rangle_{H}} \\
& =\frac{\left\langle\left[Y\left(l_{1}\right) \cdots Y\left(l_{n}\right) \mathcal{S}_{H_{0}}^{0}(\beta) \mathcal{E}(\beta, \xi)\right]_{+}\right\rangle_{H_{0}}}{\left\langle\left[\mathcal{S}_{H_{0}}^{0}(\beta) \mathcal{E}(\beta, \xi)\right]_{+}\right\rangle_{H_{0}}} .
\end{aligned}
$$

The last equality follows from Eqs. (A17) and (A20) and it is the basis for an expansion of this GF in a series of cumulants with $V=0$ but $\xi \neq 0$ : in this case the last expression in Eq. (A27) coincides with the " $\xi$ averages" defined in Eq. (3.12). The cumulants for nonzero $V$ and $\xi$ are defined through their generating function $\ln \mathcal{Z}(\beta, \xi)$,

$$
\left\langle\left[\hat{Y}\left(l_{1}\right) \cdots \hat{Y}\left(l_{n}\right)\right]_{+}\right\rangle_{c}^{V \xi}=\frac{\delta^{n} \ln [\mathcal{Z}(\beta, \xi)]}{\delta \xi\left(l_{1}\right) \cdots \delta \xi\left(l_{n}\right)}
$$

and this expression is also valid when $V, \xi$ or both are zero.

In general we are interested in the grand canonical ensemble, and we then have to substitute the total Hamiltonian by the $\mathcal{H}$ defined in Eq. ( 2.9$)$; the $\mathcal{Z}(\beta, \xi)$ becoming then the GPF [cf. Eq. (3.2)]. 


\section{APPENDIX B: GENERAL PROPERTY OF $\xi$ AVERAGES}

To derive theorem 3.1, one employs the following property:

Property B.1:

$$
\begin{aligned}
\left\langle[\hat{Y}(l) \hat{Y}(1) \cdots \hat{Y}(r)]_{+}\right\rangle^{V \xi} & \\
= & \left\langle[Y(l)]_{+}\right\rangle^{V \xi}\left\langle[\hat{Y}(1) \cdots \hat{Y}(r)]_{+}\right\rangle^{V \xi} \\
& +\delta(l)\left\langle[\hat{Y}(1) \cdots \hat{Y}(r)]_{+}\right\rangle^{V \xi} .
\end{aligned}
$$

The averages in this equation are those defined in Eq. (A24) and the symbol $\delta(l)$ means the functional derivative $[\delta / \delta \xi(l)]$. A proof of this property for the Ising model can be found in Ref. 4 [their Eq. (32)], and for the quantum Heisenberg model in Appendix B of Ref. 6. The external fields employed in the cumulant expansion for these systems are scalar fields, but in the PAM we have Fermi-type operators, and one has to use Grassmann external fields and take extra care with the signs. In the proofs of this appendix we assume that all the $Y(j)$ are of the Fermi type (i.e., they create or destroy a single electron), but property B.1 and theorem B.1 have been stated in such a way that they are also valid when some of the $Y(j)$ are Bose-type operators.

From Eq. (A26), the averages in Eq. (B1) take the form

$$
\left\langle[\hat{Y}(l) \hat{Y}(1) \cdots \hat{Y}(r)]_{+}\right\rangle^{V \xi}=\frac{\delta(1) \cdots \delta(r) \mathcal{Z}(\beta, \xi)}{\mathcal{Z}(\beta, \xi)}
$$

and taking $R=\mathcal{H}_{0}$ in Eq. (A25) we write

$$
\mathcal{Z}(\beta, \xi)=\operatorname{Tr}\left\{\exp \left(-\beta \mathcal{H}_{0}\right) \exp +\left(-\int_{0}^{\beta} d \tau H^{\prime}(\tau)\right)\right\}
$$

where $H^{\prime}(\tau)=H_{h}(\tau)+H_{e}(\tau, \xi)$ is $H_{h}+H_{e}(\tau)$ in the interaction representation [cf. Eqs. (2.8) and (3.1)]. To take the derivative with respect to $\xi(l)$ one has to take the Grassmann variable $\xi(l)$ just to the right of the $\delta / \delta \xi(l)$, with an eventual change of sign, and then eliminate that $\xi(l)$. In the $n$ th-order term of the expansion of Eq. (B3), this procedure gives

$$
\begin{aligned}
\delta(l) \int_{0}^{\beta} d \tau_{1} \cdots \int_{0}^{\beta} d \tau_{n}\{\operatorname{Tr} & {\left.\left[\exp \left(-\beta \mathcal{H}_{0}\right)\left[Y(1) \cdots Y(r) H^{\prime}\left(\tau_{1}\right) \cdots H^{\prime}\left(\tau_{n}\right)\right]_{+}\right]\right\} } \\
& =-n \int_{0}^{\beta} d \tau_{1} \cdots \int_{0}^{\beta} d \tau_{n-1}\left\{\operatorname{Tr}\left[\exp \left(-\beta \mathcal{H}_{0}\right)\left[Y(l) Y(1) \cdots Y(r) H^{\prime}\left(\tau_{1}\right) \cdots H^{\prime}\left(\tau_{n-1}\right)\right]_{+}\right]\right\}
\end{aligned}
$$

and there is an $n$ in the right-hand side (rhs) because there are $n$ factors $H^{\prime}\left(\tau_{j}\right)$. A renaming of dummy variables was necessary to obtain Eq. (B4), and the minus sign comes from the definition of $H_{e}(\xi)$.

To use this relation we employ Eq. (A27) to write

$$
\left\langle[\hat{Y}(1) \cdots \hat{Y}(s)]_{+}\right\rangle^{V \xi}=\frac{\operatorname{Tr}\left\{\exp \left(-\beta \mathcal{H}_{0}\right)\left[Y(1) \cdots Y(s) \exp +\left\{-\int_{0}^{\beta} d \tau H^{\prime}(\tau)\right\}\right]_{+}\right\}}{\mathcal{Z}(\beta, \xi)} .
$$

We expand the ordered exponential in both numerator and denominator of the rhs of Eq. (B5) and then apply the $\delta(l)$ to both sides. Employing Eq. (B4) we obtain

$$
\delta(l)\left\langle[\hat{Y}(1) \cdots \hat{Y}(r)]_{+}\right\rangle^{V \xi}=\left\langle[\hat{Y}(l) \hat{Y}(1) \cdots \hat{Y}(r)]_{+}\right\rangle^{V \xi}-\left\langle[Y(l)]_{+}\right\rangle^{V \xi}\left\langle[\hat{Y}(1) \cdots \hat{Y}(r)]_{+}\right\rangle^{V \xi},
$$

which gives Eq. (B1).

From Eq. (A28) we have

$$
\left\langle[Y(1)]_{+}\right\rangle_{c}^{V \xi}=\left\langle[Y(1)]_{+}\right\rangle^{V \xi}
$$

and repeated use of Eqs. (A28) and (B6) gives in a systematic way all the higher order cumulants

$$
\left\langle\left[\hat{Y}\left(l_{1}\right) \cdots \hat{Y}\left(l_{r}\right)\right]_{+}\right\rangle_{c}^{V \xi} .
$$

Proof of theorem 3.1. We discuss now the first part of theorem 3.1, and leave the determination of sign (the part written in italics in Sec. III) for a later consideration. To show that for any $r$ the average in the left-hand side of Eq. (B2) is "equal to the sum of products of cumulant correlation in which each term corresponds to a partition of the arguments $(1,2, \ldots, r)$, and every possible partition appears once and only once," we proceed by complete induction. The theorem is obviously true for $r=1$ [cf. Eq. (B7)], and it remains to show that if it is true for $r$ operators $Y$, it is also true for $r+1$ operators.

From Eq. (B1) it is clear that the expansion of $\left\langle[\hat{Y}(l) \hat{Y}(1) \cdots \hat{Y}(r)]_{+}\right\rangle^{V \xi}$ (with $r+1$ operators) is equal to the sum of two type of terms:

(1) Those obtained by applying $\delta(l)$ to all the terms in the expansion of $\left\langle[\hat{Y}(1) \cdots \hat{Y}(r)]_{+}\right\rangle^{V \xi}$.

(2) Those obtained by multiplying $\left\langle[Y(l)]_{+}\right\rangle^{V \xi}$ times each 
of the terms in the expansion of $\left\langle[\hat{Y}(1) \cdots \hat{Y}(r)]_{+}\right\rangle^{V \xi}$.

It is now straightforward to show that if the theorem is true for $r$ operators, all the cumulants obtained by (1) and (2) satisfy the first part of theorem 3.1, i.e., that all the partitions of $(l, 1, \ldots, r)$ are present and that each partition is present only once.

To discuss the second part of theorem 3.1, which deals with the sign of the different terms, note that in Eq. (B1) the $\hat{Y}(l)$ appears in the leftmost position in the three terms. If that position were altered in any cumulant, it would be necessary to multiply that term into a sign determined by the parity of the corresponding permutation. In the process of building up all the terms (product of cumulants) indicated by the first part of theorem 3.1, it would be necessary to apply $\delta(l)$ to the product of several cumulants. A typical case would be

$$
\begin{aligned}
\delta(l) & \langle\hat{Y}(1)\rangle_{c}\langle\hat{Y}(2)\rangle_{c} \\
& =\langle\hat{Y}(l) \hat{Y}(1)\rangle_{c}\langle\hat{Y}(2)\rangle_{c} \pm\langle\hat{Y}(1)\rangle_{c}\langle\hat{Y}(l) \hat{Y}(2)\rangle_{c},
\end{aligned}
$$

where one still has to decide which is the sign of the second term.

To follow the rules of taking derivatives with respect to Grassmann variables, we should exchange the two cumulants before we take the derivative in the second term, and one is then tempted to do that without any restriction. Nevertheless, we show below that there is a change of sign associated to the exchange of two cumulants when both of them have an odd number of $Y$ operators of the Fermi type, and from this property the second part of theorem 3.1 follows naturally.

Note that when $\xi=0$, all the cumulants have an even number of Fermi-type $Y$ operators, and they can be exchanged freely without requiring a change of sign. We still have to prove theorem B.1.

Theorem B.1. There is a change of sign associated to the exchange of two cumulants or of two simple averages, when both of them have an odd number of $Y$ operators of the Fermi type.

To prove this theorem for simple averages, we expand the time ordered exponential in Eqs. (B3) and (B5). In the $n$ th-order term of Eq. (B5) it appears

$$
\operatorname{Tr}\left\{\exp \left(-\beta H_{0}\right)\left[Y(1) \cdots Y(r) H^{\prime}\left(\tau_{1}\right) \cdots H^{\prime}\left(\tau_{n}\right)\right]_{+}\right\},
$$

which is clearly a polynomial of order $n$ in the Grassmann variables $\xi(1) \cdots \xi(n)$. We notice now that the trace in Eq. (B10) vanishes when there is an odd number of Fermi-type operators in $Y(1) \cdots Y(r)$. This property is valid because $H_{0}$ conserves the number of particles and all the $Y(j)$ either create or destroy one fermion.

We then conclude that Eq. (B10) is a polynomial of Grassmann variables $\xi(j)$ that contains only powers of the $\xi(j)$ with the same parity as the number $r$ of operators $Y_{1} \cdots Y_{r}$. As a consequence, $\mathcal{Z}(\beta, \xi)$ contains only even powers of the $\xi(j)$, and theorem B.1 is then true when applied to the simple averages of Eq. (B2).

To complete the proof for cumulants, we notice that $\left\langle[\hat{Y}(1) \cdots \hat{Y}(r)]_{+}\right\rangle_{c}^{V \xi}$ is equal to a sum of products of simple averages, such that in each term the total number of $Y$ operators that are spread in the several averages is equal to $r$, so that theorem B.1 is proved.

The phrasing of theorems 3.1 and B.1 is such that they are also valid when some of the $Y(l)$ operators are of the Bose-type, and the extension of the proof to this more general situation is straightforward.

\section{APPENDIX C: THE SIGN OF THE CONTRIBUTION OF A GRAPH}

Here we discuss the sign that must be given to the contribution of a graph, establishing different rules for $\xi=0$ versus $\xi \neq 0$. The rules for drawing the graphs that appear in the calculation of the averages $\left\langle\left[Y\left(l_{1}\right), \cdots Y\left(l_{n}\right)\right]_{+}\right\rangle^{\xi}$ are presented in rules 3.3 and 3.4, while rules 3.1 and 3.2 are for drawing vacuum graphs. In item 4 of those rules, the Fermi-type lines runming to each vertex were paired in an arbitrary way, leaving one Fermi-type edge unpaired at that vertex when their number was odd. Several open and closed loops were formed in this way, and a definite sense was arbitrarily assigned to each of them: we call this direction the "sense of the loop." When $\xi=0$ all the open loops must have two external vertices, but when $\xi \neq 0$ the open loops can also have only one external vertex or none of them, because there might be an odd number of edges arriving at a vertex. In the following discussion we consider only Fermi-type operators, because the position of the Bose-type operators does not affect the sign of the contribution. We shall then mean Fermi-type operator when we say "operator" in the remainder of this appendix. It is now convenient to introduce two concepts that shall be useful in the present computation.

Definition C.1. A graph is in a "perfect ordering" when the following relations are satisfied.

(1) For all the open loops, $\tau$ increases in all the vertices of the loop in the sense of the loop.

(2) For every closed loop, $\tau$ increases in the sense of the loop for all the vertices but one [it is impossible to satisfy (1) for a closed loop].

(3) All the $\tau$ in a given loop are either smaller or greater than all the $\tau$ in all the other loops of the graph.

There are many ways to choose a perfect ordering of a graph, but the particular choice is not important provided that we use always the same one after it has been chosen.

Definition C.2. Several Fermi-type operators of a graph contribution are in a "perfect order" when:

(1) The $Y$ operators are written from right to left following the perfect ordering we have chosen for their graph.

(2) For the two operators of each internal edge (they have the same $\tau$ ) we write the $X$ operator to the left of the $C$ operator.

We now discuss separate sign rules for $\xi=0$ and $\xi \neq 0$.

The sign of a graph when $\xi \neq 0$. In this case, the presence of an odd number of lines running to some 
edges makes it difficult to extend the rules that Hubbard ${ }^{2}$ gave for $\xi=0$, and we shall therefore give rules that are simpler to state but less systematic to apply than Hubbard's. We shall consider explicitly the graphs for $\left\langle[\hat{Y}(1) \cdots \hat{Y}(r)]_{+}\right\rangle_{H}$, i.e., a GF with $r$ external operators $\hat{Y}(1) \cdots \hat{Y}(r)$, but the rule given below is also valid for vacuum graphs. The $n$ th-order term of this GF contains the average

$$
\left\langle\left(\hat{Y}(1) \cdots \hat{Y}(r)\left[H^{\prime}\left(\tau_{1}\right) \cdots H^{\prime}\left(\tau_{n}\right)\right]\right)_{+}\right\rangle_{H}
$$

and the application of theorem 3.1 to this equation gives all the $n$ th-order graphs.

Rule C.1. To obtain the sign associated to a given graph, multiply the parities of the following two permutations.

(1) It takes the operators from the order used to write Eq. (C1) into the perfect order.

(2) It takes the operators from the perfect order into the order in which they appear in the final expression that gives the graph contribution.

As the operators $H_{h}$ are of the Bose type and can be moved freely inside the ordered parentheses in Eq. (C1), it is necessary to consider in the first step only the permutation that takes the external Fermi-type operators to their perfect order. This procedure is just the application of theorem 3.1 in two steps, and the only reason to proceed in this way is that the perfect order of the $Y$ operators in a graph provides a reference frame to organize the calculation.

The sign of the graph when $\xi=0$. In this case we shall give rules with the same labels employed by Hubbard, because of their similarity. For $\xi=0$ there is only an even number of lines running into each vertex, and for any $\mathrm{CV}$ this number is two. This simplifies the treatment, and the first step is the same step (1) employed in the $\xi \neq 0$ case: this is just rule " $\mathrm{d}$ " of Hubbard.

To calculate the change of sign that corresponds to step (2) of the $\xi \neq 0$ we proceed in three steps.

First we consider all the open loops that pass through each vertex, and note that in the perfect order, the $X$ operator is to the left of the $C$ operator in all the internal edges. To be able to pair operators of the same type at each vertex (otherwise the corresponding cumulant vanishes) it is necessary to change the order of these two operators (with a change of sign) when the arrow in the edge points towards the $\mathrm{CV}$. To correct for the sign missing in Eq. (3.20) one must also add a factor $\pm u$ to the $v(j, \alpha, \vec{k}, \sigma, \pm u)$ in rule $3.5 .2(\mathrm{c})$, and these two factors correspond to Hubbard's rule "b." In the present problem there are only two edges at each $\mathrm{CV}$, and when both are internal, the effect cancels out and the rule is not necessary. To prove this result, note that according to Rule $3.62\left(\mathrm{~b}^{\prime}\right)$, the cumulant

$$
\left\langle\left[Y\left(c ; \vec{k}_{s}, \sigma_{2},-u_{2}, \tau_{2}\right) Y\left(c ; \vec{k}_{s}, \sigma_{1},+u_{1}, \tau_{1}\right)\right]_{+}\right\rangle_{c}
$$

at each $\mathrm{CV}$ is already written with the $Y$ operators in the perfect order, with the $-u_{2}$ corresponding to the outgoing arrow. The contribution to rule 3.5.2(c) of the two internal edges running into the $\mathrm{CV}$ after correcting for the missing sign in Eq. (3.20) is then

$$
\left(+u_{2}\right) v\left(j_{2}, \alpha_{2}, k_{s}, \sigma_{2},+u_{2}\right)\left(-u_{1}\right) v\left(j_{2}, \alpha_{1}, k_{s}, \sigma_{1},-u_{1}\right) .
$$

As there is particle conservation, we have $u_{1}=u_{2}$, and when we multiply into the minus sign due to the exchange of the $X$ with the $C$ operators on the line with the arrow toward the CV, the overall sign is always plus. Hubbard's rule " $b$ " is therefore not necessary for all the $\mathrm{CV}$ with two internal lines.

For any CV with only one internal line (and therefore one external line also), one must multiply the $v(j, \alpha, \vec{k}, \sigma, \pm u)$ into $\pm u$ and also into -1 when the internal edge points toward the CV . This is the only effect that remains in the PAM of Hubbard's rule "b."

The discussion above fails for closed loops because $\tau$ can increase in the sense of the loop in all vertices but one. After putting all the operators in perfect order and then exchanging the $X$ operator with the $C$ operator for all the lines with arrows pointing to a $\mathrm{CV}$, the first and last operators in the resulting expression belong to the same vertex, and should therefore be brought together. These two operators are separated by an even number of Fermi operators, but bringing them together by an even permutation would still leave them against the order of the loop, i.e., the operator at the left would correspond to the edge with the arrow pointing toward the vertex. A permutation of odd parity is then necessary to put all the operators of any closed loop in perfect order, and this is Hubbard's rule "c."

After the three steps discussed above, the $Y$ operators that were in the order given by Eq. (C1) are now paired at each vertex according to the loops of the graph considered, each pair written in the sense of the loop. We shall denote with $\left(\alpha_{s}, \beta_{s}\right)$ the two indices of the $Y$ operators of each of those pairs, written already in the sense of the loop, i.e., $\beta_{s} \rightarrow \alpha_{s}$. All the pairs that correspond to a given vertex are still separated by many pairs that belong to other vertices of the graph, but it is only necessary to have an even permutation to put together all the pairs of each vertex. The pair associated to each $\mathrm{CV}$ is already in the same order of the cumulant of rule 3.6 $2\left(b^{\prime}\right)$, and only remains to consider the cumulants associated to the FV. If there are $p$ loops crossing an FV, we already have the corresponding operators in the or$\operatorname{der}\left(\alpha_{1}, \beta_{1}\right) \cdots\left(\alpha_{p}, \beta_{p}\right)$ while in the cumulant associated to that vertex by rule 3.62 (a) they are written in the or$\operatorname{der} Y\left(\gamma_{1}\right) \cdots Y\left(\gamma_{2 p}\right)$, where $\gamma_{1} \cdots \gamma_{2 p}$ corresponds to the same $\left(\alpha_{1}, \beta 1\right) \cdots\left(\alpha_{p}, \beta p\right)$ but in a different order. It is then necessary to associate to each of these cumulants a \pm given by the parity of the permutation that takes $\left(\alpha_{1}, \beta_{1}\right) \cdots\left(\alpha_{p}, \beta_{p}\right)$ into $\gamma_{1} \cdots \gamma_{2 p}$. This is Hubbard's rule "a."

It is now convenient to put together the rules for the calculation of the sign required by Rule $3.62(\mathrm{e})$.

Rule C.2. To calculate the sign of a graph with $\xi=0$, do the following.

(1) Define a perfect ordering for the graph according to definition C.1.

(2) The sign of the graph is the product of the following 
factors.

(a) When there are $p$ loops crossing an FV, denote with $\left(\alpha_{s}, \beta_{s}\right)$ the indices of the two $X$ operators of the sth loop at that vertex $(s=1, \ldots, p)$, written already in the sense of the loop (i.e., $\beta_{s} \rightarrow \alpha_{s}$ ). The $2 p$ Fermi-type operators at that FV appear in the cumulant of rule $3.62(\mathrm{a})$ in the order $Y\left(\gamma_{1}\right) \cdots Y\left(\gamma_{2 p}\right)$, where the $\gamma_{1} \cdots \gamma_{2 p}$ are the same $\left(\alpha_{1}, \beta_{1}\right) \cdots\left(\alpha_{p}, \beta_{p}\right)$ in a different order. For each FV multiply into $a \pm 1$ given by the parity of the permutation that takes $\left(\alpha_{1}, \beta_{1}\right) \cdots\left(\alpha_{p}, \beta_{p}\right)$ into $\gamma_{1}, \cdots \gamma_{2 p}$.

(b) For any CV with only one internal edge multiply the $v(j, \alpha, \vec{k}, \sigma, \pm u)$ of rule 3.62 (c) into $( \pm u)$, and also into a further -1 when the arrow of the internal edge points toward the CV.

(c) There is a factor -1 for every closed loop.

(d) If the graph is employed to calculate a GF with $r$ Fermi-type operators written in the order $\hat{Y}(1) \cdots \hat{Y}(r)$, multiply into a sign given by the parity of the permutation that takes $[Y(1) \cdots Y(r)]$ into the same operators written in the perfect ordering chosen for the graph. This item does not apply to vacuum graphs.

\section{APPENDIX D: COUNTING GRAPHS AND THE SYMMETRY FACTOR}

As discussed in Appendix $\mathrm{C}$, the $n$ th-order term of the perturbative expansion of the GF $\left\langle(\hat{Y}(1) \cdots \hat{Y}(r))_{+}\right\rangle_{H}$ contains the expression in Eq. (C1), and its contribution has the same form of Eq. (3.11) but with the $r$ external operators $Y(1) \cdots Y(r)$ included in the averages. When theorem 3.1 is applied to these averages, the $n$ th-order contribution can be associated to a family of graphs, and niany of them are disconnected and composed of several connected graphs. As in Sec. III, we label each topologically distinct connected graph with an index $\alpha$, and we use $n_{\alpha}$ to denote the number of times that the $\alpha$ graph appears in the $n$ th-order graph. It is clear that there might be several identical contributions associated to the same $n$ th-order graph, because all the $n$ ! permutations of the edges of a given graph give the same contribution. These identical contributions should be counted as different contributions every time they correspond to a different partition in cumulants. The correct number of times that a topologically distinct graph of $n$th order gives the same contribution is then

$$
n ! \prod_{\alpha=1}^{\infty} \frac{1}{n_{\alpha} ! g_{\alpha}^{n_{\alpha}}}
$$

where $g_{\alpha}$ is the symmetry factor of the connected graph $\alpha$ and is calculated using rule D.1 discussed below. To derive this result one applies the same arguments employed in Ref. 3: the factor $2^{n}$ of that reference is not present in our expression because the pair of vertices of any internal edge cannot be exchanged [cf. the definition of the coefficients of Eq. (3.9), discussed after Eq.(3.10)].

To calculate the symmetry factor $g_{\alpha}$ it is enough to adapt the rule given by Hubbard in Ref. 2, Appendix B . The calculation seenis rather obvious in siniple cases, but it is convenient to give the rule to deal with the more complicated ones.

Definition D.1. A vertex is said to be "internal" when all the lines running to it are internal lines.

In the PAM, only Fermi lines can run into an internal vertex, because of the form of the interaction [cf. Eq. (3.9)].

Rule D.1. To calculate the symmetry factor $g$ of a connected graph with $p_{f}$ and $p_{c}$ vertices $\mathrm{FV}$ and $\mathrm{CV}$, respectively, do the following.

(i) Number the FV with $1,2, \ldots, p_{f}$ and the CV with $1,2, \ldots, p_{c}$ so that $1,2, \ldots, q_{f}$ correspond to all the internal $\mathrm{FV}$ and $1,2, \ldots, q_{\mathrm{c}}$ to all the internal $\mathrm{CV}$.

(ii) Form the $p_{f} \times p_{c}$ matrix $N$, with elements $N_{i, j}$, where $N_{i, j}$ is the number of Fermi edges joining the FV $i$ to the $\mathrm{CV} j$.

(iii) Let $g_{1}$ be the order of the group of permutations $\mathcal{P}_{1}$ of the $q_{f} \times q_{c}$ ordered pairs $(i, j)$, which has the property that if any permutation of $\mathcal{P}_{1}$ is applied to the indices $i=1,2, \ldots, q_{f}$ and $j=1,2, \ldots, q_{c}$ of the matrix $N$, this matrix is left unchanged.

(iv) The symmetry factor is then

$$
g=g_{1} \prod_{i=1}^{q_{f}} \prod_{j=1}^{q_{c}}\left(N_{i, j} !\right)
$$

${ }^{1}$ J. Hubbard, Proc. R. Soc. London Ser. A 276, 238 (1964); 277, 237 (1964); 281, 401 (1964): these are the first three papers of a series of six.

2 J. Hubbard, Proc. R. Soc. London Ser. A 296, 82 (1966).

${ }^{3}$ M. Wortis, in Phase Transitions and Critical Phenomena, edited by C. Domb and M. S. Green (Academic, London, 1974), Vol. 3, p. 113. This volume also reviews several other types of series expansion.

${ }^{4}$ G. Horwitz and H. B. Callen, Phys. Rev. 124, 1757 (1961).

${ }^{5}$ R. Englert, Phys. Rev. 129, 567 (1963).

${ }^{6}$ R. B. Stinchcombe, G. Horwitz, F. Englert, and R. Brout, Phys. Rev. 130, 155 (1963).

${ }^{7}$ W. Metzner, Phys. Rev. B 43, 8549 (1991).

${ }^{8}$ Yu. Yzyumov, B. M. Letfulov, E. V. Shipitsyn, M. Bartkowiak, and K. A. Chao, Phys. Rev. B 46, 697 (1992).
${ }^{9}$ L. Craco, M.Sc. thesis, Federal University of Rio Grande do Sul (UFRGS), Porto Alegre RGS, Brazil, 1991.

${ }^{10} \mathrm{~A}$ few recent review articles on the subject are $\mathrm{P}$. Fulde, Solid State Phys. 41, 1 (1988); P. Schlottmann, Phys.Rep. 181, 1 (1989), G. Czycholl, ibid. 143, 278 (1986); P. A. Lee, T. M. Rice, J. W. Serene, L. J. Sham, and J. W. Wilkins, Comments Condens. Matter Phys. 12, 99 (1986).

${ }^{11}$ R. Kubo, J. Phys. Soc. (Jpn.) 17, 1100 (1962).

${ }^{12}$ A. C. Hewson, J. Phys. C 10, 4973 (1977).

13 G. G. Martinez, Ph.D. thesis, State University of Campinas (UNICAMP) Campinas, SP, Brazil, 1989.

14 J. W. Negele and H. Orland, Quantum Many-Particle Systems (Addison-Wesley, New York, 1988), Chap. 2.

${ }^{15}$ M. S. Figueira, Ph.D. thesis, State University of Campinas (UNICAMP), Campinas, SP, Brazil, 1994. 
${ }^{16}$ A. D. Anokhin and V. Yu Irkhin, Phys. Status Solidi B 165, 129 (1991); A. D. Anokhin, V. Yu Irkhin, and M. I. Katsnelson, J. Phys. Condens. Matter 3, 1475 (1991).

${ }^{17}$ The circuit C2 is the usual path in the complex plane that surrounds the real axis at a very small distance.

${ }^{18}$ H. Keiter and J. C. Kimball, Int. J. Magn. 1, 233 (1971).

${ }^{19}$ N. Grewe and H. Keiter, Phys. Rev. B 24, 4420 (1981).

${ }^{20} \mathrm{H}$. Keiter and G. Morandi, Phys. Rep. 109, 227 (1984).

${ }^{21}$ N. Grewe, T. Pruschke, and H. Keiter, Z. Phys. B 71, 75
(1988).

${ }^{22}$ T. V. Ramakrishnan and K. Sur, Phys. Rev. B 26, 1798 (1982).

${ }^{23}$ N. Grewe, Z. Phys. B 53, 271 (1983).

${ }^{24}$ Y. Kuramoto, Z. Phys. B 53, 37 (1983).

${ }^{25}$ T. K. Lee and F. C. Zhang, Phys. Rev. B 30, 1556 (1984).

${ }^{26}$ N. Grewe, Z. Phys. B 52, 193 (1983).

${ }^{27}$ N. Grewe, Z. Phys. B 67, 323 (1987). 